\title{
Extended-range predictability of sudden stratospheric warming events suggested by mode decomposition
}

\author{
Zheng Wu ${ }^{1}$, Bernat Jiménez-Esteve ${ }^{1}$, Raphaël de Fondeville ${ }^{2}$, Enikő Székely², Guillaume Obozinski ${ }^{2}$, \\ William T. Ball ${ }^{3}$, and Daniela I.V. Domeisen ${ }^{1}$ \\ ${ }^{1}$ Institute for Atmospheric and Climate Science, ETH Zürich, Switzerland \\ ${ }^{2}$ Swiss Data Science Center, ETH Zürich and EPFL, Switzerland \\ ${ }^{3}$ Department of Geoscience and Remote Sensing, Faculty of Civil Engineering and Geosciences, TU Delft, the Netherlands \\ Correspondence: Zheng Wu (zheng.wu@env.ethz.ch)
}

\begin{abstract}
Major sudden stratospheric warmings (SSWs) are extreme wintertime circulation events of the Arctic stratosphere that are accompanied by a breakdown of the polar vortex and are considered an important source of predictability of tropospheric weather on subseasonal to seasonal time scales over the Northern Hemisphere mid- and high- latitudes. However, SSWs themselves are difficult to forecast, with a predictability limit of around one to two weeks. The predictability limit for determining the type of event, i.e., wave-1 or wave- 2 events, is even shorter. Here we analyze the dynamics of the vortex breakdown and look for early signs of the vortex deceleration process with lead times beyond the current predictability limit of SSWs. To this end, we employ a mode decomposition analysis to analyze potential vorticity (PV) equation on the $850 \mathrm{~K}$ isentropic surface by decomposing each term in the PV equation using the empirical orthogonal functions of the PV. The first principal component (PC) is an indicator of the strength of the polar vortex and starts to increase from around 25 days before the onset of SSWs, indicating a deceleration of the polar vortex. We then use a budget analysis based on the mode decomposition to characterize the contribution of the linear and the nonlinear PV advection terms to the rate of change (tendency) of the first PC. The linear PV advection is the main contributor to the PC tendency at 15 to 25 days before the onset of both types of SSW events. The nonlinear PV advection becomes important between 1 to 15 days before the onset of wave- 2 events, while the linear PV advection continues to be the main contributor for wave-1 events. By linking the PV advection to the PV flux, we find that the linear PV flux is important for both types of SSWs from 15 to 25 days before the events but with different wave-2 spatial patterns, while the nonlinear PV flux displays a wave-3 wave pattern, which finally leads to a split of the polar vortex. The signals found here indicate that both the lead times for predicting the SSW onset and the lead times for predicting the type of the SSW event could potentially be extended beyond the current predictability limit of one to two weeks.
\end{abstract}

\section{Introduction} stratosphere that are accompanied by a breakdown of the polar vortex, which is a vortex of strong circumpolar westerly 
https://doi.org/10.5194/wcd-2021-14

Preprint. Discussion started: 11 March 2021

(C) Author(s) 2021. CC BY 4.0 License.

winds in the stratosphere that form in fall and decay in spring. During a major SSW event the zonal-mean zonal wind in the stratosphere reverses in mid-winter from westerly to easterly, accompanied by a sudden and dramatic increase of temperatures in the entire polar stratosphere (Labitzke, 1981). SSWs are caused by the interaction between planetary waves and the mean flow in the stratosphere (Matsuno, 1971; McIntyre, 1982). These planetary waves are generated in the troposphere by mean flow over mountains, land-sea thermal contrast, and nonlinear synoptic scale wave-wave interactions (Charney and Eliassen, 1949; Scinocca and Haynes, 1998; Held et al., 2002; Domeisen and Plumb, 2012). The waves can propagate upward into the stratosphere if their wave number is sufficiently small (e.g., wave-1 and wave-2 components) and if the background zonalmean zonal wind is eastward relative to the zonal phase speed of the waves (Charney and Drazin, 1961). When these planetary waves reach a critical level in the stratosphere, they break and deposit easterly momentum into the mean flow, resulting in a deceleration of the mean flow, which can eventually lead to a breakdown of the polar vortex, i.e., an SSW event. After an SSW event, the stratospheric anomalies can propagate downward to the lower stratosphere and influence the tropospheric weather for up to two months after the onset of events (Baldwin and Dunkerton, 2001; Kidston et al., 2015). For example, SSWs are found to be associated with an anomalously negative phase of the North Atlantic Oscillation (Domeisen, 2019) and an equatorward shift of the tropospheric extratropical jet streams (Baldwin and Dunkerton, 2001; Limpasuvan et al., 2004). The shift of the jet is crucial for the weather over North America and Europe, as it can lead to a larger probability of cold air outbreaks (Kolstad et al., 2010; King et al., 2019). Therefore, SSWs are thought to be an important source of predictability on subseasonal to seasonal (S2S) time scales over the Northern Hemisphere (NH) mid- and high latitudes (Mukougawa et al., 2009; Scaife et al., 2016; Karpechko et al., 2017). Improving the predictability of SSW events may therefore help to enhance the forecast skill in the troposphere (Sigmond et al., 2013; Domeisen et al., 2020a). On the other hand, the predictability limit for SSW events in state-of-the-art sub-seasonal prediction systems is around one to two weeks (Domeisen et al., 2020b).

Even though the polar vortex undergoes deceleration and disruption during all major SSW events, there are large differences amongst SSW events in terms of their dynamical evolution, vortex structure, and downward impact on the troposphere. Based on the geometry of the polar vortex at the onset of the event, SSWs can be classified into two types: 1) vortex displacement events, when the vortex is shifted off the pole, and 2) vortex split events, when the vortex is split into two parts (Charlton and Polvani, 2007). While displacement events are mainly attributed to the enhanced upward propagation of wavenumber 1 (hereafter: wave-1), split events are often related to strong wavenumber 2 (hereafter: wave-2) (Nakagawa and Yamazaki, 2006). Major SSWs occur about every two winters in observations but with high decadal variability (Domeisen, 2019), amongst which the frequency of split and displacement events is roughly the same (Butler et al., 2015). However, if SSWs are classified based on the zonal wavenumber of the wave flux in the lower stratosphere, there are more wave-1 events than wave- 2 events as not all split SSWs are dominated by wave-2 wave flux (Bancalá et al., 2012; Ayarzagüena et al., 2019). Split events tend to be less predictable than displacement events, especially at lead times of 1-2 weeks (Taguchi, 2018; Domeisen et al., 2020b). Given the fact that the development of the two types of SSW events are considered to be different (Matthewman et al., 2009; Albers and Birner, 2014), the dynamical processes that lead to the breakdown of the polar vortex should be distinct between displacement (wave-1) and split (wave-2) events and should also be distinguishable from normal winter days. Therefore, understanding the 
https://doi.org/10.5194/wcd-2021-14

Preprint. Discussion started: 11 March 2021

(c) Author(s) 2021. CC BY 4.0 License.

(c) (i)

Weather and

Climate Dynamics

Discussions

dynamics of the vortex disruption and identifying signals that contribute to the vortex deceleration are crucial for improving the predictability of SSWs and of each type of event, and ultimately, of the weather at the Earth's surface.

Since the stratospheric circulation is well described by Ertel's potential vorticity (PV) (McIntyre, 1982), the evolution of the polar vortex can be captured by the changes in the values and structure of PV in the stratosphere when SSWs occur. As discussed above, while the polar vortex undergoes breakdown in each major SSW event, the associated vortex structures are different for the two types of SSWs. Decomposing the PV into an empirical orthogonal function (EOF) basis, we can extract the PV structure that best describes the weakening of the polar vortex and subsequently investigate how its corresponding principal component (PC) changes with time. By projecting the other variables from PV equation (i.e., the zonal wind and meridional wind) onto the EOF basis from PV, one can analyze the contribution of each term of the equation to the change of the first PC time series in order to identify the dynamical processes that are the most relevant to the weakening of the polar vortex. This method which was proposed by Aikawa et al. (2019) is called mode decomposition analysis. The authors applied this method to diagnose the atmospheric blocking development in the Eastern Pacific and Central Atlantic, and demonstrated that the blocking index can be faithfully reconstructed using only the first 10 EOF modes. The vorticity equation was then decomposed into three terms (i.e., linear advection, nonlinear mode-to-mode interaction, and dissipation), and their contribution to the combined time evolution of the first 10 PC time series was subsequently investigated. Their results showed that the nonlinear interaction terms contribute to the increase in the amplitude of the blocking index in both regions (Eastern Pacific and Central Atlantic), but with different contributions for each of them. Since each term in the vorticity equation is represented by a linear combination of modes that correspond to specific spatial patterns and PCs, this method allowed the identification of the wind and vorticity patterns that are crucial for the development of the blocking. As the results from Aikawa et al. (2019) indicate the effectiveness of mode decomposition analysis in studying dynamically-driven events, we use the same method to study the dynamical development of SSWs, which are also driven by wave dynamics (e.g., Matsuno, 1971). Indeed, we find that the vortex weakening can be represented by the evolution of the EOF modes based on PV, and therefore, we employ the analysis for PV equation in the stratosphere to quantify the respective contribution of each EOF mode to the dynamical processes that lead to the deceleration of the polar vortex and the subsequent onset of SSWs.

80 The onset of SSW events is not just associated with the anomalously large excitation of wave activity in the troposphere (Matsuno, 1971; Polvani and Waugh, 2004; Lindgren et al., 2018), but also the stratospheric mean state and stratospheric wave anomalies prior to SSWs (Hitchcock and Haynes, 2016; Jucker, 2016; Birner and Albers, 2017; De La Cámara et al., 2019). Besides, split and displacement SSW events exhibit distinct pre-warming evolutions (Charlton and Polvani, 2007; Matthewman et al., 2009; Bancalá et al., 2012; Albers and Birner, 2014). For example, the zonal wavenumber of the wave flux leading to the breakdown of the polar vortex can be different for the two types of SSW events (e.g., Bancalá et al., 2012). Some studies suggest that the explosive growth of wave amplitude is triggered by resonant behavior, which is different between the two types of SSW events (e.g., Esler and Matthewman, 2011; Matthewman and Esler, 2011). Albers and Birner (2014) further suggest that different effects of planetary Rossby and/or gravity waves are responsible for producing the distinct vortex preconditioning that is conducive to developing the respective split and displacement SSWs events. Given the distinct dynamical developments 
of the two types of SSWs, one should be able to observe different evolutions of PV by the mode decomposition analysis for each type of SSW event. In this study, SSWs are classified into wave-1 and wave-2 events. As some studies point out that the dynamical process for a vortex breakdown starts earlier than the current predictable lead time (Polvani and Waugh, 2004; Jucker and Reichler, 2018), one would expect to see signals indicative of the vortex breakdown appearing in the mode equation budget before the onset of the SSW. The goal of the current study is to identify signals that are distinct from normal winter days ahead of the vortex breakdown with lead times longer than two weeks, and to distinguish onset signals for wave-1 and wave- 2 events beyond the currently achieved predictable lead times.

The paper is organized as follows. Section 2 describes the data for the analyses and the methodology behind the mode decomposition equation analysis. Section 3 elucidates the results of the analysis and their implications for predictability. Section 4 further provides the physical interpretation of the signals found in the mode equation budget by linking them with wave-mean flow interactions. Conclusions are given in Section 5.

\section{Data and methodology}

\subsection{Data and EOF bases}

The two data sets used are the ERA-Interim reanalysis (Dee et al., 2011) and simulations using an intermediate complexity configuration of the Isca model (Vallis et al., 2018). In particular, we use the experiment that uses prescribed strong El Niñolike SST anomalies as described in Jiménez-Esteve and Domeisen (2019). We decide to use this particular model experiment as it produces a realistic climatology and a similar SSW frequency as in reanalysis. For ERA-Interim, we use daily mean fields of potential vorticity (PV) $P$, zonal wind $u$, and meridional wind $v$ at the $850 \mathrm{~K}$ isentropic level from $1979-2018$ with a horizontal resolution of $2.5^{\circ} \times 2.5^{\circ}$. Only data north of $30^{\circ} \mathrm{N}$ (24 latitude values by 144 longitude values leading to a total of $D=3456$ grid points) from the winter season (October to April) are included in the analysis. For the Isca model simulations, the daily vertical gradient of potential temperature $(\theta)$, zonal wind $u$, and meridional wind $v$ are interpolated to the $850 \mathrm{~K}$ isentropic surface from pressure levels. The model data contains a total of 130 years and uses a T42 horizontal resolution. The PV is computed as

$P=\left(\zeta_{\theta}+f\right)\left(-g \frac{\partial \theta}{\partial p}\right)$,

where $P$ is Rossby-Ertel's PV (Hoskins et al., 1985), $\zeta_{\theta}$ is the relative vorticity on the isentropic surface, $\theta$ is the potential temperature, $f$ is the Coriolis force, $g$ is the gravity, and $p$ is the pressure. Note that any occurrence of the term potential vorticity (or PV) in this work is referring to Rossby-Ertel's PV.

The PV data (for both the ERA-Interim reanalysis and the Isca model) is further decomposed into the daily climatology and daily anomalies with respect to the climatological seasonal cycle. The EOF modes of the PV and the corresponding PC time 
https://doi.org/10.5194/wcd-2021-14

Preprint. Discussion started: 11 March 2021

(C) Author(s) 2021. CC BY 4.0 License.

Weather and

Climate Dynamics

(c) (i)

series (later used in the mode decomposition analysis) are obtained by employing principal component analysis (PCA) on daily PV anomalies at $850 \mathrm{~K}$, as follows: First, we apply PCA to the PV data by concatenating -10 to +5 days around the onset date of all SSWs. The resulting first EOF pattern $\left(E_{1} \in \mathbb{R}^{D}\right)$ is shown in the first panel in Figure 1 , with a wavenumber-0 structure centered at the pole. We then perform a second PCA on the projection of the whole winter data (October to April) onto the subspace orthogonal to $E_{1}$, i.e., by subtracting from the winter data its projection onto $E_{1}$. PCA on this projection of winter data does not contain information of $E_{1}$ and yields a total of $D=3456$ modes $\left\{E_{2}, \ldots, E_{D+1}\right\}$ (equivalent to the number of grid points). Combining $E_{1}$ and the $D$ EOF modes forms an orthogonal basis for the whole winter data, referred to as the combined set of basis vectors. Figure 1 shows the spatial pattern of the first 10 EOF modes that explain together $\approx 71 \%$ of the variance of the PV anomalies of all winter days in ERA-Interim. The respective spatial pattern of the first 10 EOF modes of PV anomalies in the Isca model data is shown in Figure C1 in Appendix C. In ERA-Interim, the variance explained by $E_{1}$ $(15.8 \%)$ for the whole winter data is slightly smaller than that of $E_{2}(16.8 \%)$, as shown in Figure 1 . These EOF modes satisfy the following relationship

$\left\langle E_{m}, E_{n}\right\rangle=\int_{\Omega} E_{m} E_{n} d x=\delta_{m n}, \quad m, n=1, \ldots, D+1$,

where $\delta_{m n}$ is the Kronecker delta function, and $\Omega$ is the region poleward of $30^{\circ} \mathrm{N}$. The PC time series of all winter days associated with $E_{1}$ (Figure 2) is highly correlated with the polar-cap averaged temperature at $30 \mathrm{hPa}$, which is a good indicator for SSWs (Blume et al., 2012). Therefore, the evolution of the first PC enables us to better understand the vortex breakdown process.
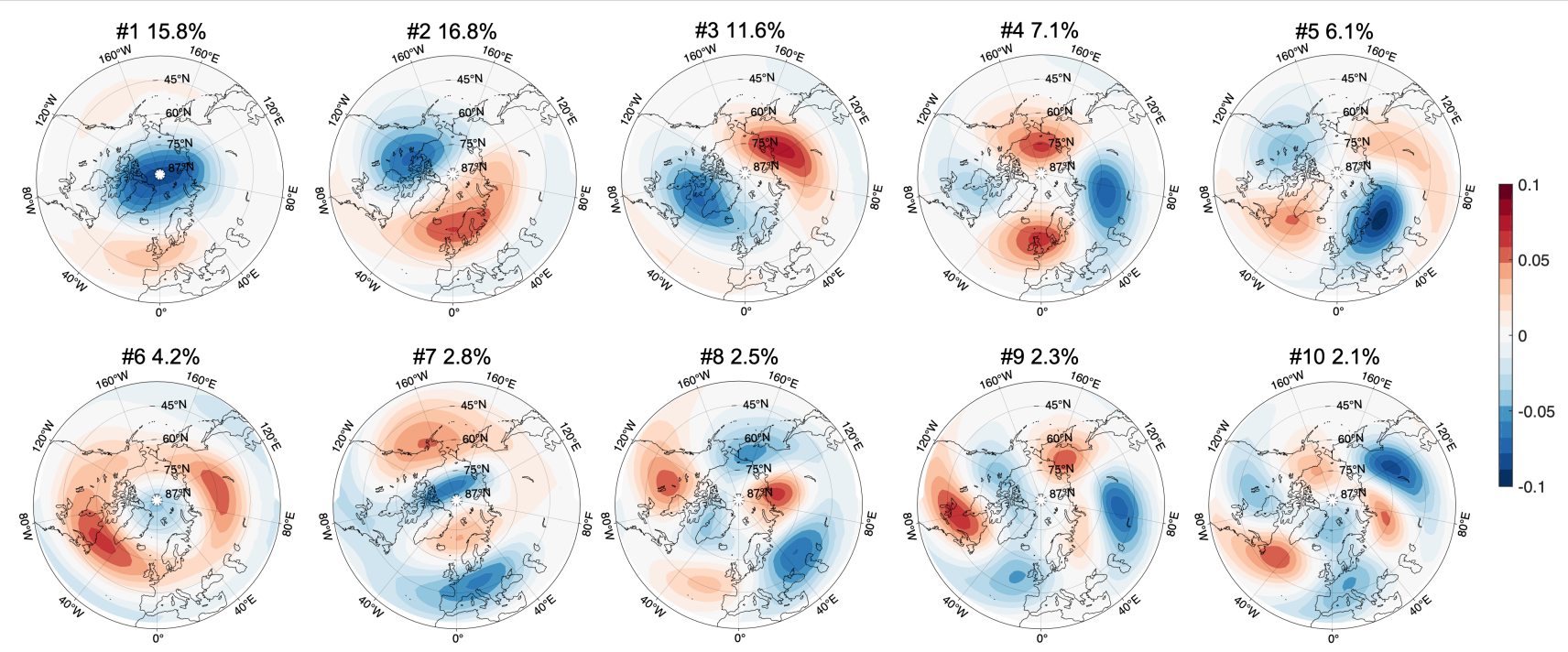

Figure 1. The first $10 \mathrm{EOF}$ spatial patterns of $\mathrm{PV}$ at $850 K\left(E_{1}, E_{2}, \ldots, E_{10}\right)$ of the combined set of basis vectors as described in the text using ERA-Interim daily data. The percentage number indicates the variance explained by each EOF. 
https://doi.org/10.5194/wcd-2021-14

Preprint. Discussion started: 11 March 2021

(C) Author(s) 2021. CC BY 4.0 License.

Weather and

Climate Dynamics

Discussions

L.

\subsection{Mode decomposition analysis}

Following the methodology from Aikawa et al. (2019), the mode decomposition analysis is applied to the PV conservation equation to study the dynamical development of SSW events. We introduce the anomalous PV equation on an isentropic surface by separating the daily anomalies from the daily climatological mean for 1979-2018 as

$\frac{\partial P_{a}}{\partial t}+\boldsymbol{V}_{c} \cdot \nabla P_{a}+\boldsymbol{V}_{a} \cdot \nabla P_{c}+\boldsymbol{V}_{a} \cdot \nabla P_{a}=F_{a}$,

where $P$ is the PV, $V$ is the wind vector field, and $F$ is the forcing term (computed here as residual). The subscript $c$ refers to the daily climatology, and $a$ refers to the daily anomalies. The anomalous PV tendency $\left(\frac{\partial P_{a}}{\partial t}\right)$ equals the sum of linear effects that consist of the advection of daily anomalous PV by climatological wind vector $\left(\boldsymbol{V}_{c} \cdot \nabla P_{a}\right)$ and the advection of climatological PV by the anomalous wind vector $\left(V_{a} \cdot \nabla P_{c}\right)$, and the nonlinear effects of the advection of anomalous PV by the anomalous wind vector $\left(\boldsymbol{V}_{a} \cdot \nabla P_{a}\right)$.

Here, we focus only on the interval between -50 to -1 days prior to the onset day of the SSW event, and concatenate the data from all SSW events for the mode decomposition analysis. We project the concatenated PV field onto the first $d=1000$ modes of the combined set of basis vectors $\left\{E_{1}, E_{2}, \ldots, E_{d}\right\}$ and get the corresponding PC time series, denoted as $\left\{A_{1}, A_{2}, \ldots, A_{d}\right\}$. We found that the first $d=1000$ modes are sufficient to reproduce the actual rate of change of the PC that is introduced below. The $\mathrm{PV}$ daily anomalies $\left(P_{a}\right)$ can be expressed as the linear combination of $\left\{E_{1}, E_{2}, \ldots, E_{d}\right\}$ with coefficients $\left\{A_{1}, A_{2}, \ldots, A_{d}\right\}$. For the wind vector daily anomalies $\left(\boldsymbol{V}_{a}\right)$, the temporal evolution of PV is correlated with that of the wind fields. Therefore, it is possible to obtain a set of spatial patterns $\left\{U_{1}, U_{2}, \ldots, U_{d}\right\}$ for $V_{a}$ by regressing $V_{a}$ onto the PC time series of PV $\left(\left\{A_{1}, A_{2}, \ldots, A_{d}\right\}\right)$. Note that the spatial patterns $\left\{\boldsymbol{U}_{1}, \boldsymbol{U}_{2}, \ldots, \boldsymbol{U}_{d}\right\}$ are not orthogonal as they are not obtained through the classical EOF mode decomposition. The $V_{\boldsymbol{a}}$ can then be represented as the linear combination of $\boldsymbol{U}_{1}, \boldsymbol{U}_{2}, \ldots, \boldsymbol{U}_{d}$ with coefficients $A_{1}, A_{2}, \ldots, A_{d}$. Then, by substituting the projection PC-expressions of $P_{a}$ and $\boldsymbol{V}_{a}$ into Eq. (3) and taking the inner product between Eq. (3) and one specific EOF mode $E_{k}$, we obtain the mode equation budget of the rate of change (or tendency) of $A_{k}$ as (detailed derivation in Appendix A)

$$
\frac{d A_{k}}{d t}=\frac{1}{C_{k}}\left(-\sum_{n=1}^{d} L_{k n}^{A} A_{n}-\sum_{n=1}^{d} L_{k n}^{B} A_{n}-\sum_{m=1}^{d} \sum_{n=1}^{d} N_{k m n} A_{m} A_{n}+F_{k}\right),
$$

where $C_{k}=\left\langle A_{k}, A_{k}\right\rangle$ is the eigenvalue of mode $k ; L_{k n}^{A}=\left\langle E_{k}, \boldsymbol{V}_{\boldsymbol{c}} \cdot \nabla E_{n}\right\rangle$ and $L_{k n}^{B}=\left\langle E_{k}, \boldsymbol{U}_{n} \cdot \nabla P_{c}\right\rangle$ are the inner products between the linear advection terms $\left(\boldsymbol{V}_{c} \cdot \nabla E_{n}\right.$ and $\left.\boldsymbol{U}_{n} \cdot \nabla P_{c}\right)$ and $E_{k} ; N_{k m n}=\left\langle E_{k}, \boldsymbol{U}_{m} \cdot \nabla E_{n}\right\rangle$ is the inner product between the nonlinear advection term $\left(\boldsymbol{U}_{m} \cdot \nabla E_{n}\right)$ and $E_{k}$; and $F_{k}=\left\langle E_{k}, F_{a}\right\rangle$ is the residual term. The two linear advection terms are combined together as the total linear advection. Using Eq. (4), we then compute the contribution of each mode to the linear and nonlinear advection terms and thus to the total rate of change of $A_{k}$ to determine which modes (or combinations of modes) play an important role in identifying signals representative of SSWs from normal winter days.

From the power spectrum of each PC time series $A_{k}$ (not shown), the power of $A_{1}$ to $A_{25}$ is concentrated at periods longer than one week, which is different from the power spectrum of the other PCs beyond $A_{25}$. Therefore, based on these power spectra, 
here we consider the associated modes $E_{1}$ to $E_{25}$ as low modes, which together explain around $85 \%$ of the total variance, and modes beyond $E_{25}$ as high modes. To separate the contributions from low- and high EOF modes, the summation over all modes from equation Eq. (4) is divided into the summation of low modes and that of high modes. Thus, Eq. (4) can be written as

$$
\begin{aligned}
\frac{d A_{k}}{d t}=\frac{1}{C_{k}}( & -\sum_{n=1}^{l}\left(L_{k n}^{A}+L_{k n}^{B}\right) A_{n}-\sum_{n=l+1}^{d}\left(L_{k n}^{A}+L_{k n}^{B}\right) A_{n} \\
& \left.\quad-\sum_{m=1}^{l} \sum_{n=1}^{l} N_{k m n} A_{m} A_{n}-\sum_{m=1}^{l} \sum_{n=l+1}^{d}\left(N_{k m n}+N_{k n m}\right) A_{m} A_{n}-\sum_{m=l+1}^{d} \sum_{n=l+1}^{d} N_{k m n} A_{m} A_{n}+F_{k}\right) \\
= & \frac{1}{C_{k}}\left(L_{\text {low }}+L_{\text {high }}+N_{\text {low-low }}+N_{\text {low }- \text { high }}+N_{\text {high }- \text { high }}+F_{k}\right),
\end{aligned}
$$

where $l=25$ represents the $n$th mode that is treated as the last low mode; $L_{l o w}$ and $L_{\text {high }}$ are the linear low- and high mode contributions, respectively, and $N_{\text {low-low }}, N_{\text {low-high }}$; and $N_{\text {high-high }}$ are the contributions from nonlinear low-low, lowhigh, and high-high mode interactions, respectively. We performed sensitivity tests for a range of $l$ values, and our results and conclusions are robust for values of $l>25$. Moreover, the contribution from modes higher than $l=25$ is very small (not shown). In this study, we only focus on the tendency of the first PC time series $\frac{d A_{1}}{d t}(k=1)$ due to the strong relationship between $A_{1}$ and SSWs.

\subsection{SSW definition and PV flux}

The major SSW definition follows the criterion of Charlton and Polvani (2007), based on the reversal of the daily zonal-mean zonal winds at $10 \mathrm{hPa}$ and $60^{\circ} \mathrm{N}$, and a return to westerlies afterward for at least 10 consecutive days before the final warming. Two events in the same season are treated as distinct SSWs if they are separated by at least 20 days. The central dates of split and displacement SSW events in ERA-Interim before 2014 are taken from Karpechko et al. (2017) with 11 split events and 12 displacement events. In addition, we added the SSW event in 2018, which is classified as split event based on the vortex geometry (Charlton and Polvani, 2007). The definition of wave-1 and wave-2 SSW events is based on the eddy heat flux at 100 $\mathrm{hPa}$ and $60^{\circ} \mathrm{N}$ similar to Bancalá et al. (2012). If the wave- 2 component of eddy heat flux is larger than the wave- 1 component by $15 \mathrm{Kms}^{-1}$ in the period of -2 to 0 days before the SSW event for at least 1 day, then the SSW event is classified as a wave-2 event, otherwise as a wave-1 event. Note that the time window around the SSW event (day -2 to day 0) that is used to classify the type of event is shorter than that in Bancalá et al. (2012). The reason for using a shorter window is to reduce the overlap between the time interval used to define the type of SSW event and the lead times that emerge as relevant in the predictability of wave-1 vs. wave- 2 events. According to this definition, there are 18 wave- 1 and 7 wave- 2 SSW events in ERA-Interim. Note that not all split events are dominated by wave- 2 wave flux (only 6 out of 12 split SSWs are also classified as wave- 2 events), while one displacement event is dominated by wave- 2 wave flux according to this definition. 
In order to provide a physical interpretation for each term in Eq. (4) and understand the physical process that can lead to the disruption of the polar vortex, we introduce the poleward flux of PV on an isentropic surface given by Tung (1986) as

$\nabla \cdot \mathcal{F}=\left[\rho_{\theta}\right]\left[\left(\rho_{\theta} P^{* *}\right) v^{*}\right] \cos \phi$

where $\mathcal{F}$ is the Eliassen-Palm flux (EP flux), $P$ is the potential vorticity, $v$ is the meridional wind, $\rho_{\theta}$ is density in isentropic coordinates, defined as $\rho_{\theta}=-\frac{1}{g} \frac{\partial p}{\partial \theta}$, and $\phi$ is the latitude. The brackets denote the zonal mean, one asterisk denotes the deviation from the zonal mean, and two asterisks denote the deviation from the density-weighted zonal average, as in

$P^{* *}=P-\frac{\left[\rho_{\theta} P\right]}{\left[\rho_{\theta}\right]}$

The formulation of PV flux in Eq. (6) is also equivalent to that defined using $v^{* *}$, as in Eq. (4.5) in Tung (1986). The left-hand term in Eq. (6) is the EP flux pseudo-divergence and the right-hand term in Eq. (6) is the zonal-mean northward flux of PV on the isentropic surface. According to Tung (1986), the PV flux corresponds to the pseudo-divergence of the EP flux along isentropic surfaces and acts as the net eddy forcing term of the mean flow. Note that the pseudo-divergence of the EP flux is used here (instead of the divergence) due to the fact that the density on isentropic surfaces changes with time, which is the main difference from the conventional EP flux (Edmon et al., 1981).

To better illustrate the physical interpretation of the individual terms in equation Eq. (4), using the concepts of PV flux and EP flux pseudo-divergence, we rewrite Eq. (3) as

$210 \frac{\partial P_{a}}{\partial t}+\frac{1}{\rho_{\theta}} \nabla \cdot\left(\rho_{\theta} P_{a} \boldsymbol{V}_{c}\right)+\frac{1}{\rho_{\theta}} \nabla \cdot\left(\rho_{\theta} P_{c} \boldsymbol{V}_{a}\right)+\frac{1}{\rho_{\theta}} \nabla \cdot\left(\rho_{\theta} P_{a} \boldsymbol{V}_{a}\right)+P\left(\frac{1}{\rho_{\theta}} \frac{\partial \rho_{\theta}}{\partial t}\right)=F_{a}$.

The second to fourth terms on the left-hand side are the linear and nonlinear terms of the density weighted PV flux divergence, while the last term is the local density tendency. Since density weighted PV is proportional to vorticity, the second to fourth terms can be interpreted as the dynamical contribution to the PV evolution. On the other hand, since the density tendency is inversely proportional to the temperature tendency, the fifth term can be interpreted as the thermodynamic component of the PV evolution. Eq. (8) is derived by converting the advection terms in Eq. (3) into density weighted flux divergence using the continuity equation. Taking the inner product between Eq. (8) and $E_{1}$ and neglecting the longitudinal variation of $E_{1}$ given its wavenumber- 0 structure, i.e., $E_{1} \approx E_{1}(\phi)$, we obtain an approximated expression of the linear and nonlinear terms in equation Eq. (8) (a detailed derivation is provided in Appendix B):

$L_{\text {low }}+L_{\text {high }} \approx 2 \pi a^{2} \int_{\phi_{1}}^{\phi_{2}} E_{1}(\phi) \frac{\partial\left[\rho_{\theta} P_{a}^{* *} v_{c}^{*}\right]+\left[\rho_{\theta} P_{c}^{* *} v_{a}^{*}\right]}{\partial \phi} \cos \phi d \phi$,
$N_{\text {low-low }}+N_{\text {low-high }}+N_{\text {high-high }} \approx 2 \pi a^{2} \int_{\phi_{1}}^{\phi_{2}} E_{1}(\phi) \frac{\partial\left[\rho_{\theta} P_{a}^{* *} v_{a}^{*}\right]}{\partial \phi} \cos \phi d \phi$,

where $a$ is the radius of the Earth, $\phi$ is the latitude with $\phi_{1}=30^{\circ} \mathrm{N}$ and $\phi_{2}=90^{\circ} \mathrm{N}$. Next, we combine the mode equation budget from Eq. (4) with the zonal-mean PV flux from Eq. (6). Based on the relation shown in Eq. (6) and the fact that $\nabla \cdot \mathcal{F} \propto \frac{\partial[u]}{\partial t}$ 
from the zonal momentum equation, one can further obtain the following relation

$-\frac{\partial\left[P^{* *} v^{*}\right]}{\partial \phi} \propto-\frac{\partial}{\partial \phi}\left(\frac{\partial[u]}{\partial t}\right) \propto \frac{\partial \zeta_{\theta}}{\partial t}$.

From Eq. (10), the meridional gradient of zonal-mean PV flux $\left(-\frac{\partial\left[P^{* *} v^{*}\right]}{\partial \phi}\right)$ connects to the vorticity tendency $\left(\frac{\partial \zeta_{\theta}}{\partial t}\right)$, which is the dynamical component of the rate of change of $A_{1}$.

\section{Mode equation budget}

As shown in Figure 1, the first EOF spatial pattern $E_{1}$ of the PV daily anomalies in ERA-Interim takes the shape of a wavenumber-0 structure with a negative anomaly at the pole. Thus, a positive (negative) value of $A_{1}$ (PC time series of $E_{1}$ ) indicates a weakening (strengthening) of the polar vortex. For example, Figure 2a shows the corresponding $A_{1}$ for all winter days of ERA-Interim (8490 days in total) and the red line indicates the onset day of an SSW event. Before SSW events occur, $A_{1}$ increases significantly and is strongly positive on the central day, indicating a weakening and breakdown of the polar vortex. Similar EOF spatial patterns are found for the Isca model data (Figure C1), together with a similar increase in $A_{1}$ when approaching the SSW central day (Figure 2b). The Isca model data contains a total of 130 years of simulation, corresponding to 27300 winter days, of which Figure $2 \mathrm{~b}$ only shows the winter days from the first 40 years as an example. By understanding what contributes to the change of $A_{1}$, we extract information that helps to explain the breakdown of the polar vortex during SSW development. We compute the mode equation budget of $A_{1}$ using daily data concatenated for the period 50 to 1 days prior to the onset day for all SSW events. There are a total of 25 SSWs in the ERA-Interim reanalysis data, and 78 SSWs in the Isca model run. In the following, we will show the composite of SSW events for both reanalysis and model data.

\subsection{Mode equation budget for ERA-Interim}

Figure 3 shows the SSW composite of the $A_{1}$ mode decomposition budget. The first, second, and third rows show the results of the composites of all SSW events, wave-1 SSW events, and wave-2 SSW events, respectively. We apply a 5-days running mean to all lines in Figures 3-6 to remove the high-frequency fluctuations. The bold lines in Figure 3-6 indicate the values that are outside of the 2.5 th to 97.5 th percentile range of normal winter days values, which is computed via a bootstrapping procedure described as follows. In each bootstrap sample, we randomly select with replacement 25 sets of 50 consecutive non-SSW winter days (excluding the 50 days before each SSW) across the different years. We then calculate the mean of these 25 sets of non-SSW days to represent the "composite" of normal winter days. We repeat the bootstrap re-sampling procedure $B=1000$ times and compare the SSW composite against the 2.5th and 97.5th percentile of the $B$ bootstrap samples. The same procedure is applied for the two types of SSWs and for the Isca model data, using the number of SSWs in that category as the number of sets of consecutive non-SSW winter days in the bootstrapping. The reconstructed $A_{1}$ tendency (black line in Figure 3) is computed from Eq. (5). The left panels show each term in Eq. (5), while the right panels show the combined effect 
https://doi.org/10.5194/wcd-2021-14

Preprint. Discussion started: 11 March 2021

(c) Author(s) 2021. CC BY 4.0 License.

(c) (i)

Weather and

Climate Dynamics

Discussions

L

(a)

ERA-Interim

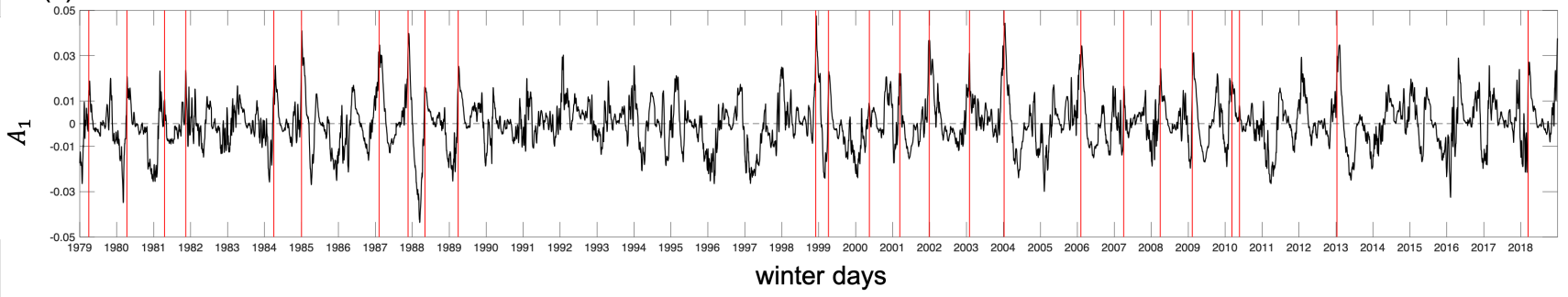

(b)

Isca-model

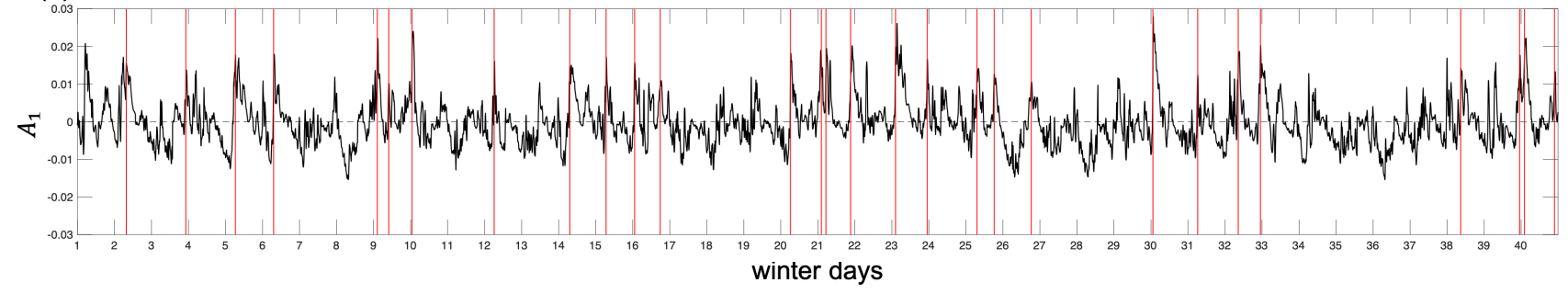

Figure 2. The PC time series $\left(A_{1}\right)$ of winter days corresponding to the first EOF spatial pattern $\left(E_{1}\right)$ using (a) ERA-Interim reanalysis data and (b) Isca model output data. In (a) all winter days are shown and in (b) only the winter days for the first 40 years are shown as an example. The PC time series for winter days of the remaining 90 years in the Isca model data can be seen in Figure C2 in Appendix C. The red vertical lines indicate the onset dates of SSW events.

of all linear (red) and all nonlinear (green) terms without separating the contributions from low- and high EOF modes. Figure 3a indicates that around 25 days before the central day of SSWs $\frac{d A_{1}}{d t}$ starts to increase, and the increase steepens around 10 days before the SSW event, leading to a large positive $A_{1}$ on the central day as shown in Figure 2a. Along with the increase of $\frac{d A_{1}}{d t}, L_{\text {low }}$ (red) also increases and it is well correlated with the $A_{1}$ tendency $(r=0.8)$. In fact, $L_{\text {low }}$ starts to increase from 35 days before the events but its effect is offset by other terms and it becomes the only contributor to the increase of $\frac{d A_{1}}{d t}$ at 25to 15-day leads. The nonlinear term $N_{\text {low-low }}$ (green) shows a rapid increase around two weeks before the SSW event and, together with the linear term $L_{l o w}$, significantly contributes to the changes in $A_{1}$. The high-frequency components are overall weaker than the low-frequency terms, especially the $N_{\text {high-high }}$ (cyan), but the $N_{\text {low-high }}$ (orange) has large variations and tends to offset the effect of $L_{l o w-l o w}$ at -25 to - 15 days before the vortex weakening.

The contributions from each term are different between the composites of wave-1 vs. wave-2 events (Figures $3 \mathrm{c}$ and $3 \mathrm{e}$ ), respectively. The amplitude of $L_{\text {high }}$ (blue) is large at around one week before the event, and the nonlinear terms are overall small for wave-1 events (Figure 3c) when compared to wave-2 events (Figure 3e). The amplitude of $N_{\text {low-low }}$ is the largest starting one week before the onset for the wave-2 events. To better illustrate the different contributions of linear and nonlinear advection terms in Eq. (5) to the increase of $\frac{d A_{1}}{d t}$ in the two types of events, we combine all the linear terms and all the nonlinear terms in Eq. (5) and show the results in the right panels of Figure 3. The linear advection term is the most important contribution 
https://doi.org/10.5194/wcd-2021-14

Preprint. Discussion started: 11 March 2021

(c) Author(s) 2021. CC BY 4.0 License.

Weather and

Climate Dynamics

Discussions

from around 25 to 15 days before both SSW types, and the nonlinear advection term becomes more dominant from day - 15 to the onset of the wave-2 SSW events (Figure 3f). On the other hand, the linear advection term plays a central role from day -25 to day 0 for the wave-1 SSWs (Figure 3d). The distinct contributions from the linear and nonlinear advection terms for wave-1 vs. wave-2 events indicate that the processes leading to the vortex breakdown of the two types of SSW events are dynamically different. The simultaneous contributions from linear and nonlinear terms in the all-SSWs composite (Fig. 3a,b) can be effectively viewed as being due to the average over wave-1 and wave-2 SSW events within the composite (Figure 3b). In both types of events, the breakdown of the polar vortex is initiated by the increase of the linear advection term, which helps to weaken the polar vortex around one month before the events and plays a central role until day -10 . Around 10 days before the event, the linear (nonlinear) advection term is the dominant contribution for the breakdown of the vortex for the wave- 1 (wave-2) events, while the nonlinear (linear) terms are less important or even counteracting the increase of $\frac{d A_{1}}{d t}$. Therefore, the relative importance of linear and nonlinear terms emerges as a good indicator for the identification of the type of SSW events with a lead time of around 10 days prior to the events.

The relative importance of the linear and nonlinear advection terms for the two types of SSW events is similar to that of the stratospheric wave amplitude of the wavenumber 1 and 2 components for wave-1 and wave-2 SSW events as shown in Bancalá et al. (2012). From their composite analysis of the wave-2 SSW events, the wave-2 component of the geopotential height anomaly in the stratosphere is significantly positive during day -10 to day 0 and the anomalous increase of wave- 1 component was found in the period from day -30 to day -10 . In Figure 3 we find that the linear and nonlinear advection terms behave similar to the wave-1 and wave- 2 components of the upward propagating wave activity. Consistent with the two periods in Bancalá et al. (2012), the evolution of linear and nonlinear terms can be separated into two different time periods, with one from day -25 to day -15 and the other from day -10 to day 0 . Since the displacement (split) SSW events are mainly attributed to the enhanced upward propagation of wave-1 (wave-2) (Nakagawa and Yamazaki, 2006; Bancalá et al., 2012), we also look into the contributions of linear and nonlinear terms to $\frac{d A_{1}}{d t}$ for displacement and split SSW events (shown in Figure D1 in Appendix D), with very similar results. Comparing Figure D1a-b with Figure 3c-d, the behavior of each term (the total linear term and nonlinear advection terms) is very similar as most of the displacement events are wave- 1 events (only the event in March 2000 is a wave-2 event). Among the 12 split SSWs, 6 events are dominated by wave-1 wave flux, and most of them do not have a clear split-type behavior as those dominated by wave- 2 wave flux. On the other hand, comparing Figure D1c-d to Figure 3e-f, the differences between linear and nonlinear terms are more obvious for wave-2 SSWs as only half of the split events are included in wave- 2 events.

In order to examine the significance in the differences and the robustness of the relative importance in the linear and nonlinear advection terms for the two types of SSW events, we perform bootstrapping on individual wave-1 and wave-2 events with replacement, respectively. We repeat the re-sampling $B=1000$ times and compute the means and the standard deviation for the sum of linear and nonlinear terms. The results of the bootstrapping are shown in Figure 4. There is almost no overlap between the \pm 1 standard deviation of wave-1 (red) and wave-2 (black) events in neither the linear advection (Figure 4a) nor the nonlinear advection (Figure $4 \mathrm{~b}$ ) terms when considering one week before the onset of the events. In particular, the 
(a)

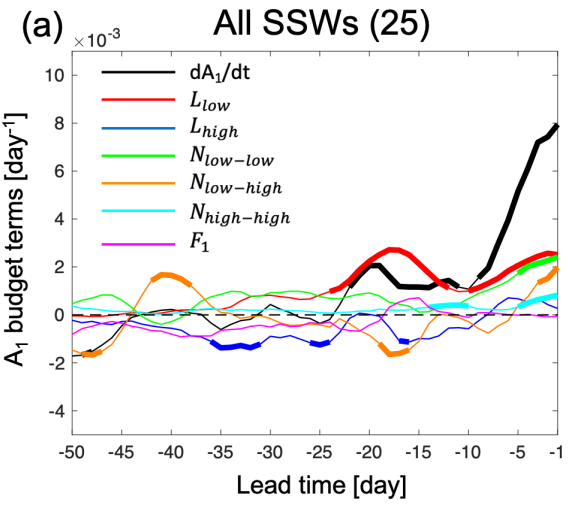

(c)
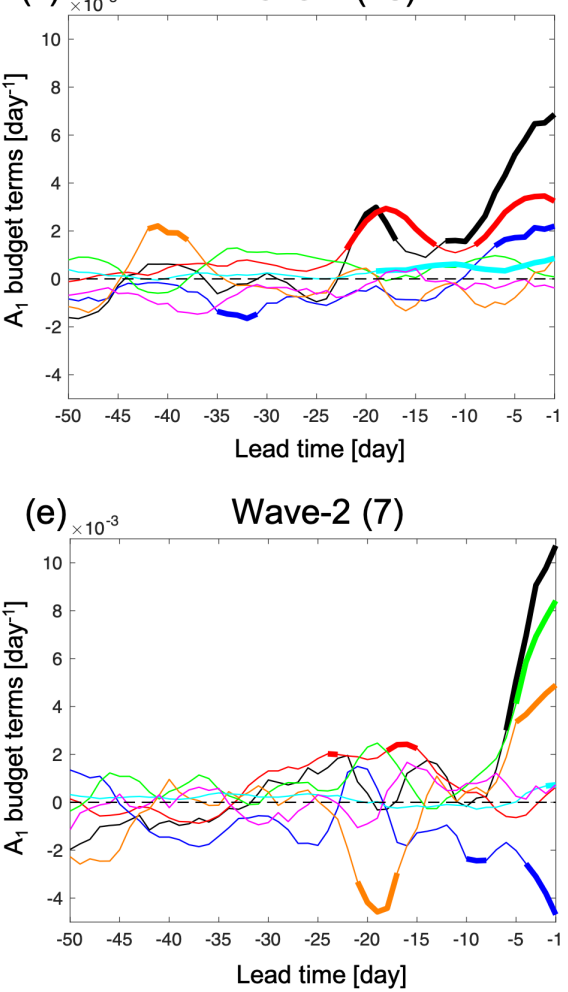

(b) $\times 10^{-3} \quad$ All SSWs (25)

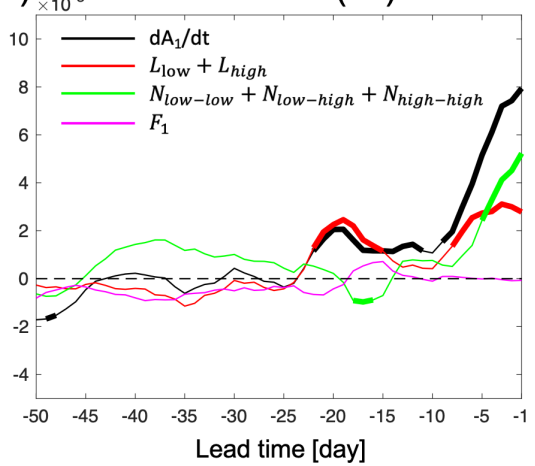

(d)

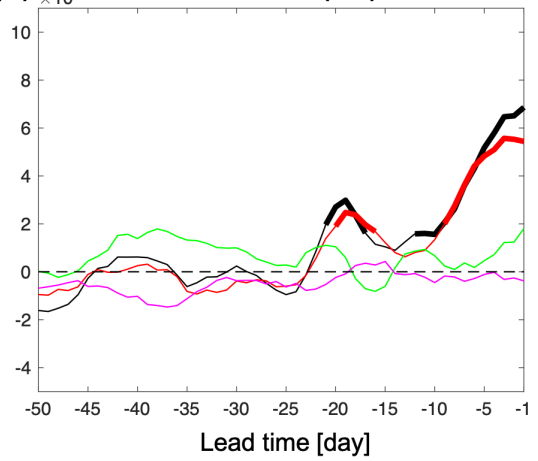

(f) $\times 10^{-3} \quad$ Wave-2 (7)

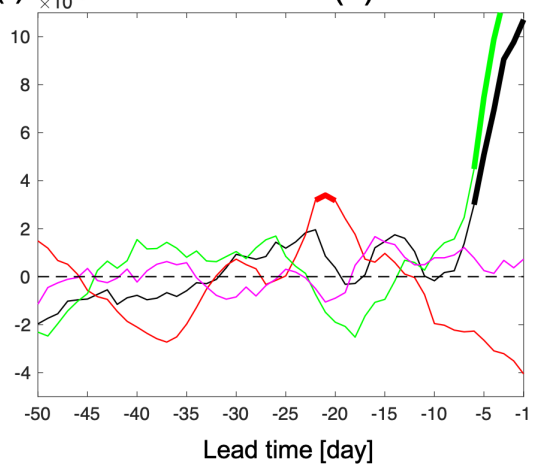

Figure 3. The composite of the $A_{1}$ budget as a function of lead time from 50 to 1 days before the onset of events in ERA-Interim. (a, b) Composite of all (25) SSW events, (c, d) composite of wave-1 events, (e, f) composite of wave-2 events. (a, c, e) show each term of the mode equation budget (Eq. (5)) separately, and (b, d, f) show the sum of the linear and nonlinear terms for the different types of events. Number in the bracket in each panel title indicates the number of SSW events. A 5-day running mean is applied to all lines. Bold lines indicate the values that are outside of the 2.5 th to 97.5 th percentile range of normal winter days values from bootstrapping as described in the text. The representation of each line color in (c,e) and in (d,f) is the same as the legend in (a) and in (b), respectively. 
separation of the wave- 1 and wave- 2 events in the linear advection is as early as 10 days before the onset of the events. Figure 4 demonstrates the significance and robustness of the differences in the contribution of the linear and nonlinear advection terms to $\frac{d A_{1}}{d t}$ in the wave-1 and wave-2 SSW events at least up to one week before the events. Another point to highlight is that significant anomalies of the linear terms are observed around 20 days before both types of SSW events, which is beyond the current predictability limit of SSWs of one-two weeks.

(a)

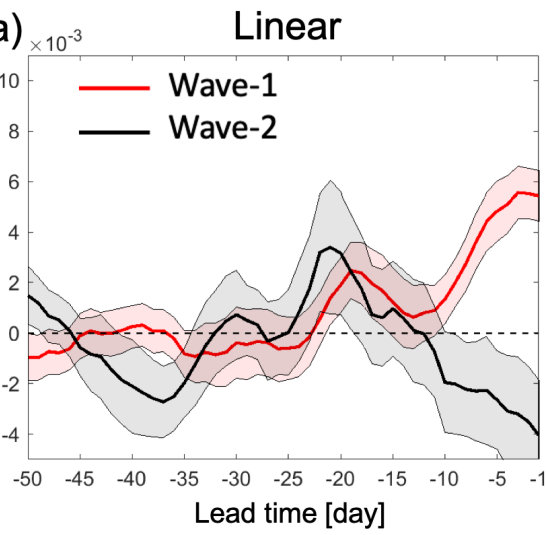

(b)

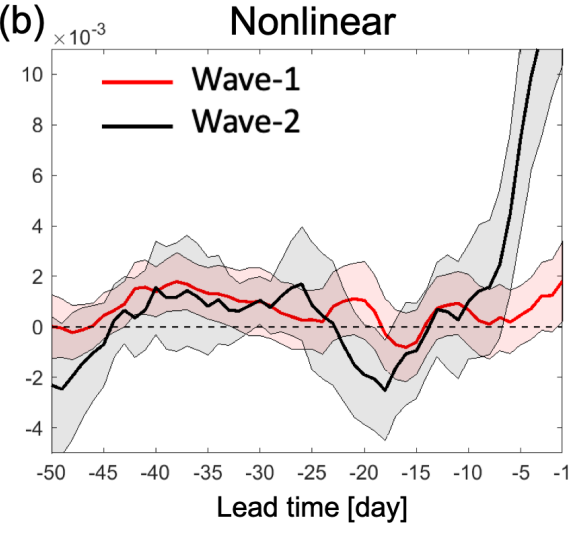

Figure 4. The comparison of the $A_{1}$ budget of the bootstrapping between wave- 1 and wave- 2 SSW events as a function of lead time from 50 to 1 days before the onset of events in ERA-Interim. (a) Sum of linear advection terms and (b) sum of nonlinear advection terms for wave-1 events (red) and wave-2 events (black). A 5-day running mean is applied to all lines. Bold lines and the shading indicate the mean and \pm 1 standard deviation of a bootstrapping using $B=1000$ samples.

\subsection{Mode equation budget for the simplified Isca model}

Given the limited number of SSW events in the reanalysis data and to further examine the characteristics and robustness of the linear and nonlinear contributions to the vortex breakdown, we now apply the same analysis to the output of the simplified model experiments. We use the method from Section 2 to derive the EOF spatial patterns and apply the mode decomposition analysis to the data concatenating 50 to 1 days prior to the $78 \mathrm{SSW}$ present in the model data. The EOF spatial patterns derived from the model output are similar to those derived from ERA-Interim, especially the first 10 EOF modes (Figure C1), indicating that the model is able to simulate the PV features as in the reanalysis.

Figure 5 shows the results of the $A_{1}$ budget for the SSW composites. Similar to the results in ERA-Interim (Figure 3), the linear term $L_{\text {low }}$ starts to increase at around day -25 , but the increase in $\frac{d A_{1}}{d t}$ starts at around day -10 (Figure 5a), which is later than that in ERA-Interim (around day -25). In the period of day -10 to day 0 of SSWs, $N_{l o w-h i g h}$ increases rapidly.

315 The increasing $N_{\text {low-high }}$ and $L_{\text {low }}$ lead to a rapid increase of $\frac{d A_{1}}{d t}$. We note that in the 2 days before the central date, the 
https://doi.org/10.5194/wcd-2021-14

Preprint. Discussion started: 11 March 2021

(c) Author(s) 2021. CC BY 4.0 License.

Weather and

Climate Dynamics

Discussions

L.

magnitudes of the wave-1 and wave- 2 wave fluxes are similar (with differences $<5 \mathrm{Kms}^{-1}$ ) in some SSW events that the model simulated. In order to make a clearer separation, we exclude events with the difference in the magnitude of wave-1 and wave-2 heat flux at $100 \mathrm{hPa}$ and $60^{\circ} \mathrm{N}$ smaller than $5 \mathrm{Kms}^{-1}$ as these events cannot be clearly categorized as either wave-1 or wave- 2 events. Therefore, in the model we have 36 wave-1, 27 wave-2, and 15 unclassified events. Figure $5 \mathrm{c}$ shows that $L_{\text {low }}$ increases and becomes distinct from normal winter days starting at a lag of 20 days for wave-1 SSWs. Different from the $A_{1}$ budget of wave-1 SSWs in ERA-Interim (Figure 3c), $N_{\text {low-high }}$ starts to increase from day -7 and becomes an important contributor to $\frac{d A_{1}}{d t}$. For wave-2 SSWs, Figure 5e shows that $N_{\text {low-high }}$ is the main contributor to the increase of $\frac{d A_{1}}{d t}$ from day -10, and $N_{\text {low-low }}$ as well as $L_{\text {high }}$ are the second largest contributor to $\frac{d A_{1}}{d t}$ from day -5 , which is different from the evolution of $L_{h i g h}$ for wave-2 SSWs in ERA-Interim (Figure 3e). In both types of events, $L_{l o w}$ starts to increase at around day -20 , which helps to weaken the polar vortex in the preconditioning stage and is similar to the evolution of $L_{\text {low }}$ (with a smaller amplitude) in the same period in ERA-Interim. The effects of all linear and all nonlinear terms combined are shown in the right panels in Figure 5. The evolution of the linear and nonlinear advection terms, and thus of $\frac{d A_{1}}{d t}$, for the Isca model (Figure $5 b$ ) is similar to the evolution of these terms for the ERA-Interim data (Figure 3b), indicating that the Isca model successfully reproduces the vortex breakdown in the 10 days preceding the SSWs. The increase of $\frac{d A_{1}}{d t}$ can therefore be used to predict the occurrence of SSWs with one-two weeks lead time. Even though the distinct increase of $\frac{d A_{1}}{d t}$ only shows up at around day -10, the linear advection term actually increases as early as day -29 . However, this amplification in the linear term is offset by the nonlinear and forcing terms, which leads to a near-zero $\frac{d A_{1}}{d t}$. Figure $5 \mathrm{~d}$ and $5 \mathrm{f}$ show the evolution of the linear and nonlinear advection terms of the wave-1 and wave-2 SSW composites, respectively. Different from wave-1 events in ERA-Interim, the linear and nonlinear terms are equally important. However, when comparing wave- 1 with wave- 2 composites, one can still see the difference in the relative importance of linear and nonlinear terms for the two types of SSWs. The nonlinear term is stronger in wave-2 SSWs than in wave-1 SSWs and it is more than twice as large as the linear term one week before the central day (Figure 5f). Thus, the finding that the linear (nonlinear) term is important for wave-1 (wave-2) events is also true for the Isca model data. The main differences compared with the reanalysis data are that $\frac{d A_{1}}{d t}$ exhibits a substantial increase only from day -10 , and the variations of all terms in Eq. (5) are overall small before day -10, which could potentially limit the predictability of SSWs in the Isca model. Different reasons might be able to explain for the discrepancies between model and reanalysis. These include the model complexity (e.g. lack of parameterizations for gravity waves breaking and interactive ozone chemistry) and the coarse model horizontal resolution (T42), both of which might lead to an underestimation of some of the high-frequency variability in comparison with reanalysis. Another potential explanation could just be due to the larger number of SSW events in the model than in the reanalysis composite. In addition, an important difference from the reanalysis is the larger values of the nonlinear term for both types of SSWs. This later effect might be related to the stronger SSW-sensitivity to wave-2 forcing in the idealized models, i.e., most of the SSW are likely triggered by wave-2 activity (Gerber and Polvani, 2009), in contrast with wave-1 being more dominant in more complex GCMs or reanalysis datasets (see for example Figure 3 in Ayarzagüena et al., 2019). Note that even when classifying an SSW event as a wave-1 event in the model, its wave- 2 component, although weaker than the wave-1 component, might still play an important role in the evolution of the event in the model. 

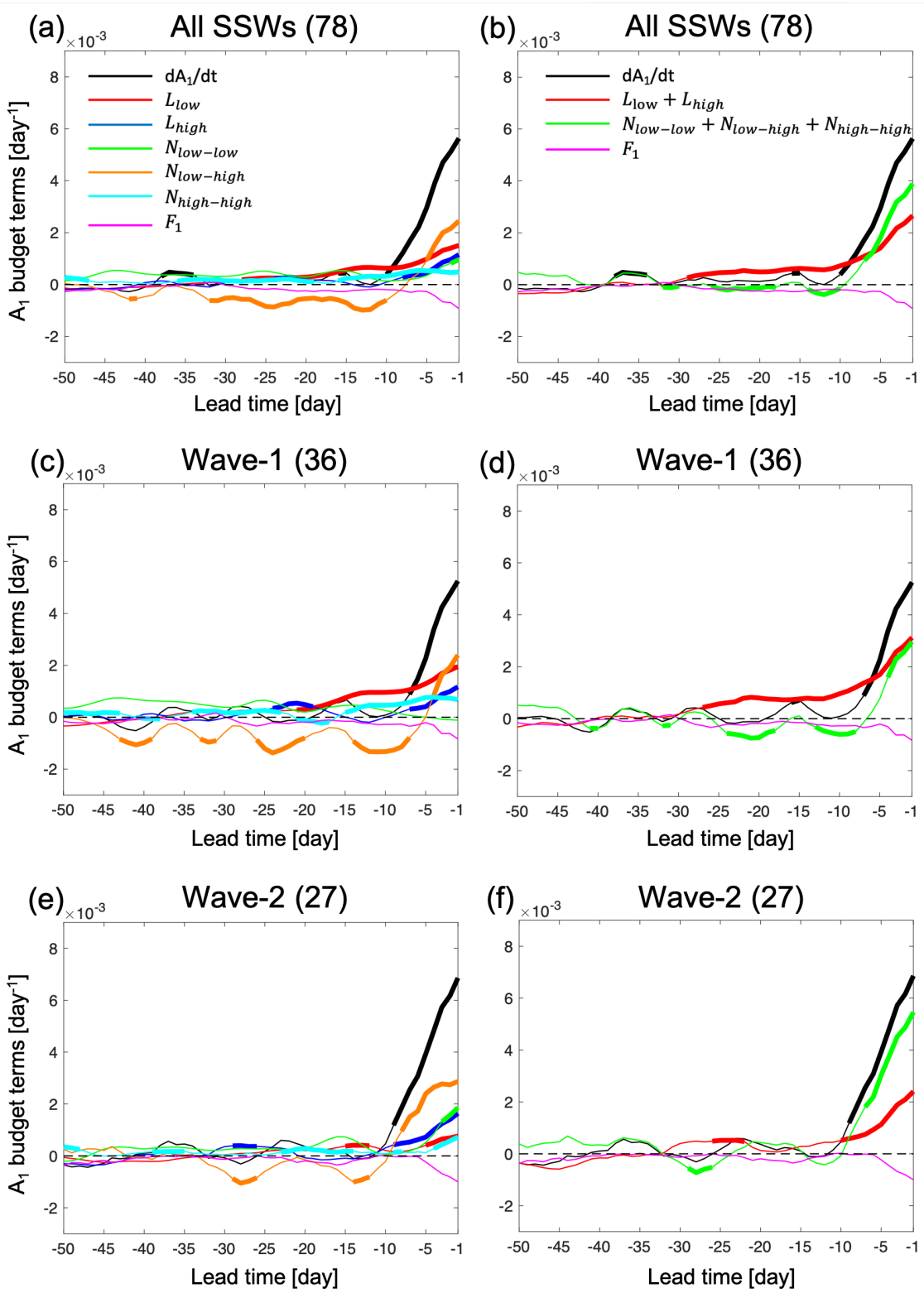

(f) $\times 10^{-3} \quad$ Wave-2 (27)

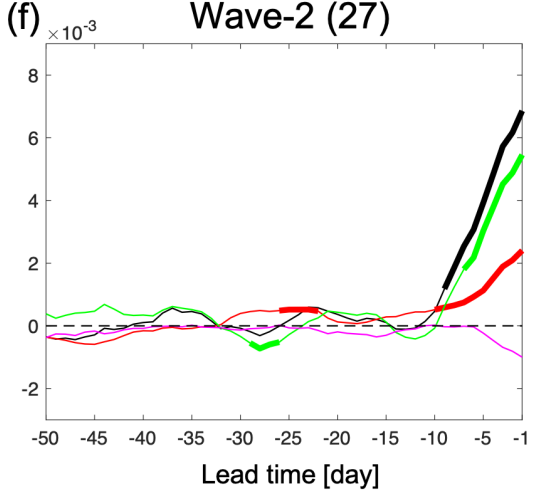

Figure 5. The composite of $A_{1}$ budget as a function of lead time from 50 to 1 days before the onset of the events in the Isca model output. (a, b) Composite of the total $78 \mathrm{SSW}$ events, (c, d) composite of 37 wave-1 events, and (e, f) composite of 26 wave-2 events. (a, c, e) show each term of Eq. (5), and (b, d, f) show the sum of all linear terms and all nonlinear terms, respectively. All lines are smoothed by a 5-day running mean. Bold lines indicate the values that are outside of the 2.5th to 97.5th percentile range of normal winter days values from bootstrapping as described in the text. The representation of each line color in (c,e) and in (d,f) is the same as the legend in (a) and in (b), respectively. 
https://doi.org/10.5194/wcd-2021-14

Preprint. Discussion started: 11 March 2021

(c) Author(s) 2021. CC BY 4.0 License.

(c) (i)

Weather and

Climate Dynamics

Discussions

\section{Physical interpretation of the mode decomposition}

In our analyses above, we found that the signals emerging during the vortex breakdown are significantly different from normal winter days, and also different between wave- 1 and wave- 2 events. We also observed that the signals that are characteristic of SSWs emerge as early as 20-25 days before the onset of SSWs. Given that these results hint at the potential for improved predictability of SSWs, in this section we provide a physical interpretation of these signals in the mode decomposition budget analysis.

As introduced in Section 2, the linear and nonlinear advection terms in Eq. (4) and Eq. (5) are closely linked to the PV flux divergence, which offers a more intuitive interpretation in an Eulerian framework. In this section, we use ERA-Interim data to illustrate the physical interpretation of the increase of the linear and nonlinear advection terms in Eq. (5). The motivation to introduce the PV flux into the PV equation is that the zonal-mean PV flux is connected to the pseudo-divergence of the EP flux along isentropic surfaces, and thus acts as the net eddy forcing term of the mean flow, allowing for connections with the theory of wave-mean flow interaction (i.e., Eq. (6) and Eq. (10)). According to McIntyre and Palmer (1983), the wave activity of planetary waves is converted to the angular momentum of the mean flow, which violates the non-acceleration condition (Charney and Drazin, 1961), leading to the reversal of the mean flow. To understand the importance of the zonal-mean PV flux during the development of SSWs, we decompose the zonal-mean PV flux into different components as in Ayarzaguiena et al. (2011),

$\left[\rho_{\theta}\right]\left[\rho_{\theta} P^{* *} v^{*}\right] \cos \phi=\left[\rho_{\theta}\right]\left(\left[\rho_{\theta} P_{c}^{* *} v_{c}^{*}\right]+\left[\rho_{\theta} P_{c}^{* *} v_{a}^{*}\right]+\left[\rho_{\theta} P_{a}^{* *} v_{c}^{*}\right]+\left[\rho_{\theta} P_{a}^{* *} v_{a}^{*}\right]\right) \cos \phi$,

where the subscript $c$ represents the daily climatology, and $a$ represents the daily anomaly. On the right-hand side of Eq. (11), the first term corresponds to the climatological planetary waves, the second and third terms correspond to the interaction between the climatological planetary waves and the daily anomalies, and the fourth (last) term corresponds to the interaction between anomalies. Similar to Eq. (3), the second and third right-hand terms can be viewed as linear components and the fourth term as the nonlinear component. The first term can be seen as a constant since its variation with time is very small as shown in Figure 6. Figure 6 shows the composite of zonal-mean poleward PV flux averaged from north of $45^{\circ} \mathrm{N}$ and its decomposition in Eq. (11) as a function of lead time before SSW events. When approaching the onset day of SSWs, the zonalmean PV flux (black) becomes increasingly negative, indicating a weakening of the polar vortex. This strengthening of the negative zonal-mean PV flux magnitude is mainly due to the linear interaction between the climatological planetary waves and the daily anomalies (red) and the nonlinear interaction between anomalies (green), which correspond to linear and nonlinear PV advection terms (right columns in Figure 3), respectively. Even though the climatological planetary waves (blue) also have negative contribution to the total PV flux, the variations are very small with time. Similar to the distinct contributions of linear and nonlinear PV advection terms for wave-1 and wave-2 SSW composites, the negative total zonal-mean PV flux is mainly due to its linear component in wave-1 SSWs (red in Figure 6b) and is mainly because of its nonlinear component in wave-2 SSWs (green in Figure 6c). The nonlinear (linear) component of the PV flux even becomes positive just before the wave-1 
(wave-2) events, opposing the weakening of the polar vortex. Similar behaviours can also found in the linear advection term for wave-2 SSWs composite (Figure 3f). Even though the amplitude of the negative linear PV flux at around day -15 in wave-2 SSWs composite is small, it helps to weaken the polar vortex and offset the effect induced by the positive nonlinear PV flux.

Note that it is the meridional gradient of the poleward zonal-mean PV flux that is used to approximate the linear and nonlinear PV advection terms as demonstrated in Eq. (9). The poleward zonal-mean PV flux is proportional to the magnitude of its meridional gradient and thus, one can use it to approximate the linear and nonlinear advection terms and to provide a physical interpretation of the signals found in Section 3.
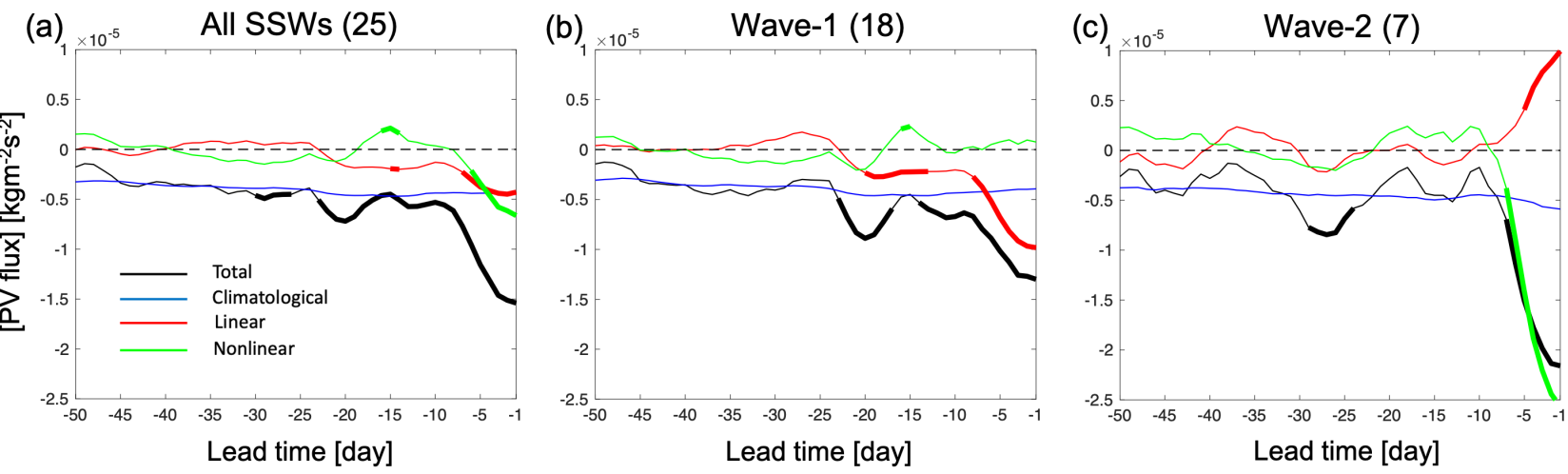

Figure 6. Composites of zonal-mean poleward PV flux averaged from north of $45^{\circ} \mathrm{N}$ and its decomposition in Eq. (11) as a function of lead time before SSWs: (a) all SSW events, (b) wave-1 events, and (c) wave-2 events in ERA-Interim. A 5-day running mean is applied to all lines. Bold lines indicate the values that are outside of the 2.5 th to 97.5 th percentile range of normal winter days values from bootstrapping as described in the text. Sub-figures (a-c) all share the same color legend as (a).

From Figure 6, the linear and nonlinear zonal-mean PV flux can be potential quantities to predict the type of SSW events. Given the abrupt change of PV flux in the 10 days preceding the onset of the events, we next examine how the spatial patterns of the poleward PV flux lead to their distinct zonal-mean contributions in the two types of SSWs. Figure 7 shows the poleward linear and nonlinear PV flux horizontal patterns of the wave-1 and wave- 2 composites. As shown in the previous analyses, the linear signals start to amplify at around 20-25 days preceding the onset of both types of SSWs, and later the nonlinear signals become the most important contributors to the breakdown of the vortex from day -10 for wave- 2 events, while the linear signals keep amplifying for wave-1 events. Thus, two different lead times (day -15 and day -5) are displayed in Figure 7 as example to present the spatial pattern of the PV flux in the two different periods before the onset of the events. The spatial patterns for the linear PV flux of wave-1 events are quasi-stationary from around day -20 (Figure 7a and 7b). A similar wave-2 pattern is also shown in linear PV flux for wave-2 events in the period of 28 to 12 days preceding the onset date (Figure 7c). This pattern disappears from day -11 (not shown), and the wave pattern shown in Figure $7 \mathrm{~d}$ develops continuously until the central day of the SSW event. At the same time the positive values of PV flux increase, leading to a positive zonal-mean PV flux when 
https://doi.org/10.5194/wcd-2021-14

Preprint. Discussion started: 11 March 2021

(c) Author(s) 2021. CC BY 4.0 License.

Weather and

Climate Dynamics

Discussions

close to day 0. Different from the linear PV flux, the nonlinear PV flux shows a clear higher wavenumber pattern in the second period of the development of wave-2 SSWs shown in Figure 7h, which has a strongly negative PV flux over western North America and a strongly positive PV flux over eastern North America. The downwind growth of the PV flux reaches its extreme magnitude over the North Atlantic and Northern Europe and then the PV flux gradually weakens downstream over North Asia and the North Pacific. This organized wave pattern in the nonlinear PV flux does not emerge until day -11 and remains largely stationary until the onset of the wave- 2 events. In the early stage of the warming (day -25 to day -15 ), the nonlinear PV flux is very weak in magnitude and not organized in structure as shown in Figure 7g. Since previous studies suggested that split SSWs have a predominantly barotropic structure (Manney et al., 1994; Matthewman et al., 2009; Albers and Birner, 2014), we investigate the vertical structure of the nonlinear PV flux in the wave-2 SSWs composite. Figure $8 \mathrm{~b}$ shows the longitude-height cross section of the nonlinear PV flux of the wave-2 SSWs composite at day -5 as an example. From Figure $8 b$, the wave pattern shown in Figure $7 \mathrm{~h}$ extends throughout the stratosphere and displays barotropic characteristics for wave- 2 events. On the other hand, the longitude-height cross section of the linear PV flux at day -5 of the wave-1 SSWs in Figure 8a displays a more baroclinic structure in the Eurasia and Pacific regions. The spatial pattern of the nonlinear PV flux in the composite of wave-1 events exhibits substantial transient fluctuations without a clear wave pattern before day -9 (Figure 7e), and shows a more stable and organized spatial pattern in the period from day -9 to day 0 (Figure 7f). However, the magnitude of the nonlinear PV flux in wave-1 events is weaker than its linear flux counterpart and weaker than the nonlinear PV flux in the wave-2 events composite.

Even though the spatial patterns of the linear PV flux in the two types of SSWs show a wave-2 pattern in the period of 20 to 10 days preceding the central day of SSWs, the locations of the maximum and minimum of the PV flux shift around $30^{\circ}$ in longitude in Pacific and North America regions (Figures 7a and 7c). In the period of day -10 to day 0, the wave pattern shown in Figure 7h sets in and leads to the final split of the vortex for wave-2 SSWs. The question is what makes the differences in the evolution of the vortex breakdown such that the linear PV flux remains important for wave- 1 events, while the nonlinear PV flux amplifies for wave-2 events. Some previous studies suggested that the pre-SSW evolution of the polar vortex is distinct between split and displacement events (Charlton and Polvani, 2007; Bancalá et al., 2012), and this preconditioning could trigger the nonlinear resonance of planetary waves in the lower stratosphere, leading to the split of the polar vortex (Albers and Birner, 2014; Boljka and Birner, 2020). Here we examine the PV and meridional wind daily and zonal anomalies $\left(P_{a}^{* *}\right.$ and $v_{a}^{*}$, respectively, see Eq. (11)) for the wave-1 and wave-2 events to understand their distinct evolutions after day -10 . Figure 9a-b (Figure 9c-d) shows the spatial pattern of $P_{a}^{* *}\left(v_{a}^{*}\right)$ at day -15 before wave-1 and wave- 2 events, respectively. Both $P_{a}^{* *}$ and $v_{a}^{*}$ present wave-1 patterns, but the positive and negative anomalies are located in different regions. The whole pattern of $P_{a}^{* *}$ in wave-2 SSWs is around $60^{\circ}$ further east compared to wave-1 SSWs. The negative $v_{a}^{*}$ is mainly located over eastern North America and the northern North Atlantic, which is important for the negative PV flux in the same region (Figure 7a) for wave-1 SSWs, while for the wave-2 SSWs the negative $v_{a}^{*}$ covers all of North America. These differences in the location of $P_{a}^{* *}$ and $v_{a}^{*}$ between the two types of SSW events are amplified from day -10 (Figure 9e-h). The magnitudes of $P_{a}^{* *}$ and $v_{a}^{*}$ in the period of day -10 to day 0 are larger than in the period of day -25 to day -15 . The negative $P_{a}^{* *}$ is located more over 
https://doi.org/10.5194/wcd-2021-14

Preprint. Discussion started: 11 March 2021

(c) Author(s) 2021. CC BY 4.0 License.

(c) (i)

Weather and

Climate Dynamics

Discussions

Northern America, while the positive $P_{a}^{* *}$ is located over the North Atlantic and Europe for wave-1 SSWs (Figure 9e). For wave-2 SSWs, the pattern of $P_{a}^{* *}$ is the opposite (Figure 9f). The positive $v_{a}^{*}$ extends to the full North Pacific (Figure 9g) and both $P_{a}^{* *}$ and $v_{a}^{*}$ maintain wave-1 structure for wave-1 SSWs. In contrast, $v_{a}^{*}$ (Figure $9 \mathrm{~h}$ ) develop a wave-2 structure from day -10 of wave- 2 events. The weak positive $P_{a}^{* *}$ over Asia (Figure 9f) further develops from day -5 , resulting in a wave-2 structure over mid-latitude at day 0 (not shown). We also find that the nonlinear PV flux and the $P_{a}^{* *}$ and $v_{a}^{*}$ form a positive feedback from around day -10 to the onset of the wave- 2 events. As the amplitude of $P_{a}^{* *}$ and $v_{a}^{*}$ becomes larger, the nonlinear PV flux is also amplified, particularly in the region of the negative nonlinear PV flux over western North America and the North Atlantic as shown in Figure 7h. The strong negative nonlinear PV flux contributes to more negative net zonal-mean PV flux values, suggesting a zonal-mean EP flux convergence. This EP flux convergence thus further decelerates the polar vortex. According to the non-acceleration theorem (Charney and Drazin, 1961), the deceleration of the polar vortex is accompanied by stronger wave activity, which is represented by the growing amplitude of $P_{a}^{* *}$ and $v_{a}^{*}$. We also note that the spatial pattern of $P_{a}^{* *}$ in Figure 9f finally leads to the split of the polar vortex in wave- 2 events, with the positive values corresponding to one of the daughter vortices located around $60^{\circ} \mathrm{W}$.

\section{Conclusions}

In this paper we employ a mode decomposition analysis to investigate the preconditioning of sudden stratospheric warming events. We study the (linear and nonlinear) terms in the potential vorticity equation by means of a budget analysis in order to identify the components in the first PC time series that allow us to distinguish the behavior of the polar vortex during SSW events from normal winter days. Moreover, we identify characteristics of SSWs that help to identify the type of event (wave1 vs. wave-2) during the dynamical development of SSWs. The mode decomposition analysis allows us to obtain a mode equation budget that describes the temporal evolution of the stratospheric dynamical processes that lead to the breakdown of the polar vortex. A better understanding of the vortex weakening process may help to improve the predictability of SSW events. We find a significant increase in the first PC time series $\left(\frac{d A_{1}}{d t}\right)$ representing the evolution of the strength of the polar vortex, which becomes increasingly positive at around 25 days before the onset of the SSW. This marks the start of the vortex weakening process and indicates an acceleration of the polar vortex breakdown. This signal is clearly distinct from the evolution of the vortex characteristics for normal winter days. The identified lead time of 25 days is far beyond the current two-weeks predictability limit of SSW events (Domeisen et al., 2020b), and could thus potentially be used to improve the predictability of SSWs. The identified increase of $\frac{d A_{1}}{d t}$ is mainly due to the increase of the linear PV advection term, which preconditions the weakening of the polar vortex. While recent work suggests that split SSW events are less predictable than displacement events (Taguchi, 2018; Domeisen et al., 2020b), the preconditioning by the increasing linear PV advection and PV flux is important for both wave-1 and wave-2 SSW events at around 20 days before the onset of the event, implying a similar predictability with 20 days lead for both types of events. From around 10 days before the events, the nonlinear PV advection term increases rapidly for wave-2 SSWs, but it remains small for wave-1 SSWs. As the nonlinear PV advection increases, the linear PV advection 
https://doi.org/10.5194/wcd-2021-14

Preprint. Discussion started: 11 March 2021

(c) Author(s) 2021. CC BY 4.0 License.

(c) (i)

Weather and

Climate Dynamics

Discussions

L

(b) Wave-1 day -5
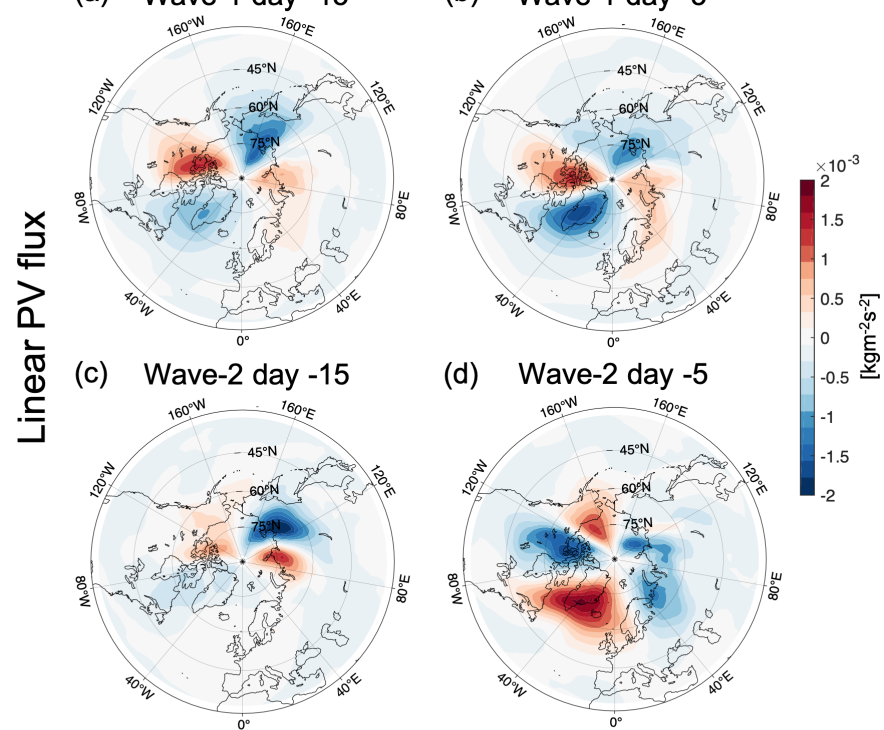

(e) Wave-1 day -15
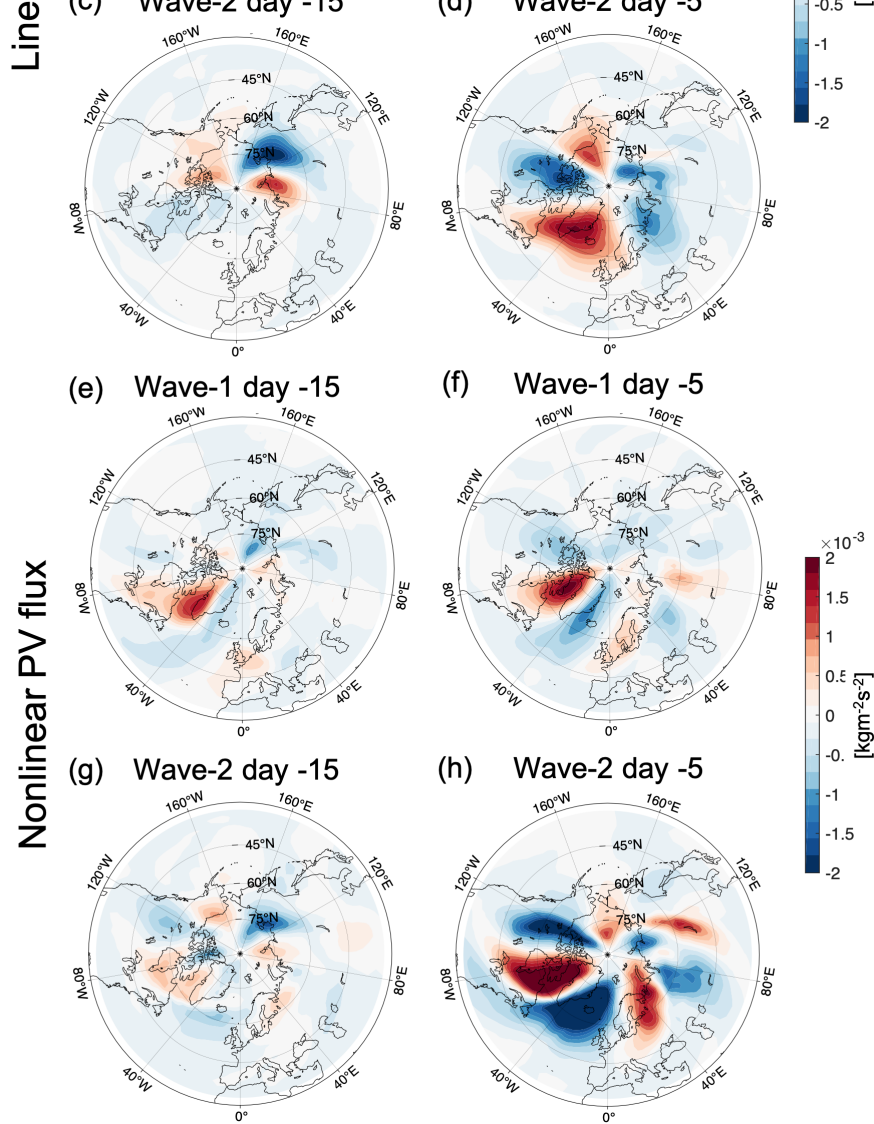

(f) Wave-1 day -5

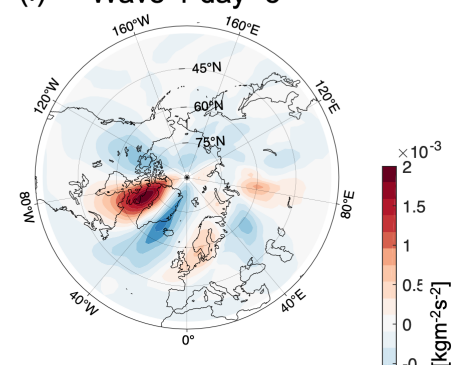

(h)

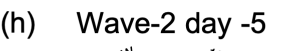



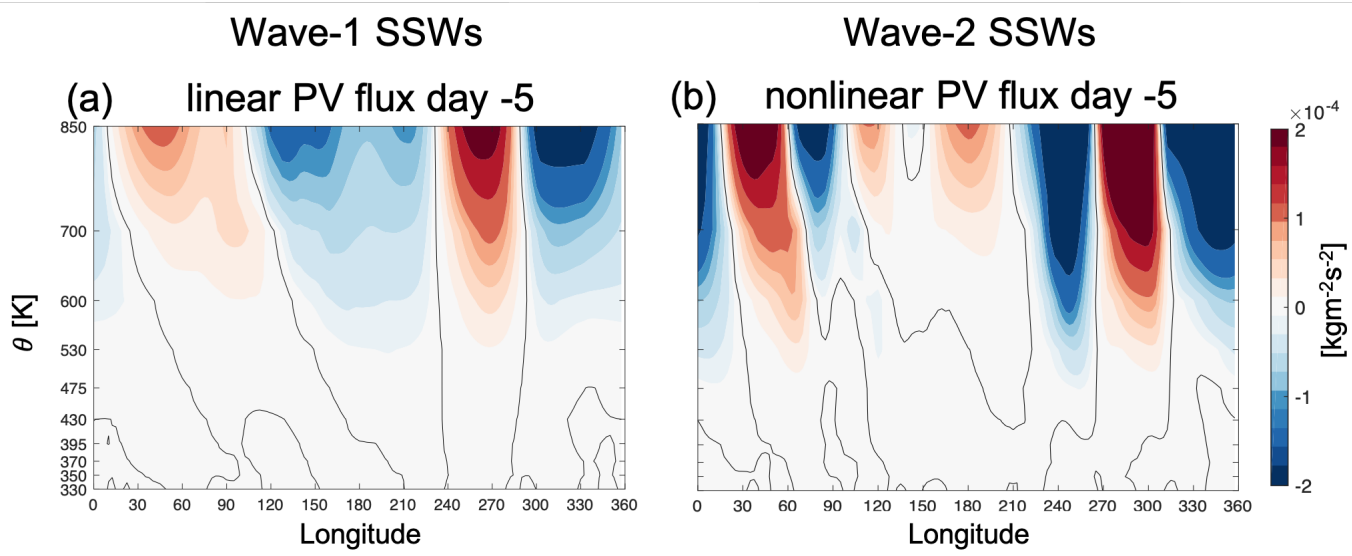

Figure 8. The longitude- $\theta$ cross section of PV flux averaged north of $45^{\circ} \mathrm{N}$ at day -5 . (a) Linear PV flux for wave- 1 SSWs and (b) nonlinear PV flux for wave-2 SSWs. Black line indicates the zero value of PV flux.

drops dramatically prior to wave-2 SSWs. The distinct behavior of the linear and nonlinear advection terms in this 10-day period suggests that the type of SSW event could be predicted at around 10 days to one week prior to the events. Note that the type of event is determined by the larger wavenumber component of eddy heat flux in the period of -2 to 0 days before the events and hence the 10-day lead times need to be interpreted with caution. On the other hand, this classification does not depend on the wave flux information in the earlier period, and the linear and nonlinear terms could be interpreted as the signals with lead times of at least one week.

Even though the contributions from linear and nonlinear PV advection terms are different in the two types of SSW events, their overall effects on $\frac{d A_{1}}{d t}$ within 10 days before the events are the same, causing $\frac{d A_{1}}{d t}$ to increase dramatically. The breakdown of the polar vortex can be divided into two periods based on the different behavior of the linear and nonlinear terms. The first period starts 25 days to two weeks before the onset when the linear term weakens the polar vortex for both types of SSWs, and the second period starts around 10 days before the onset date when the final vortex breakdown structure is determined. These two different time periods before the onset of the events are consistent with previous studies, especially for wave-2 SSWs (Labitzke, 1981; Bancalá et al., 2012; Albers and Birner, 2014), which suggested that an amplification of the wave-1 component allows the wave-2 wave flux to grow and propagate more effectively into the already weakening polar vortex region. Similar results are also found in the simplified Isca model experiments where the linear advection term increases in the first period for the preconditioning of the warming, and the relative importance of linear and nonlinear terms can also be seen in the two types of SSWs, confirming the robustness of the findings from the ERA-Interim reanalysis data.

In both the ERA-Interim reanalysis and the simplified Isca model experiments, the increase of $\frac{d A_{1}}{d t}$ is more abrupt for wave-2 SSW events than for wave-1 events and thus results in a larger $\frac{d A_{1}}{d t}$ in the 10-day period preceding the onset of the events. The abrupt changes in $\frac{d A_{1}}{d t}$ are mainly due to the exponential increase in the nonlinear PV advection. By contrast, the linear PV 
https://doi.org/10.5194/wcd-2021-14

Preprint. Discussion started: 11 March 2021

(C) Author(s) 2021. CC BY 4.0 License.
Weather and

Climate Dynamics

Discussions

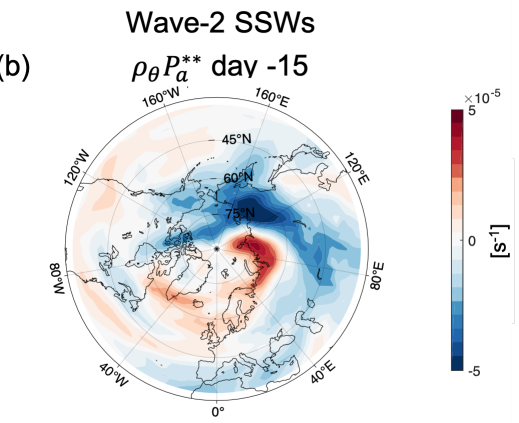

(d)

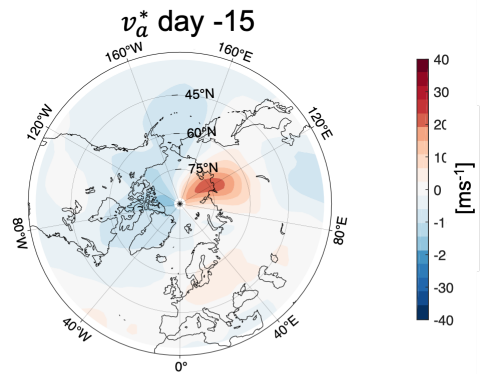

(f)

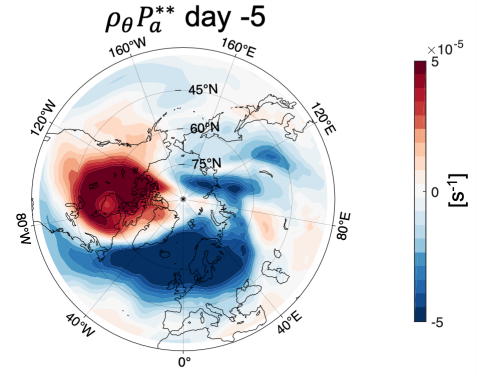

(h)

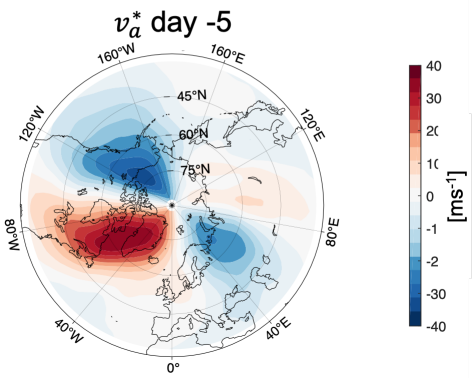

Figure 9. The spatial pattern of variables after removing the daily climatology and zonal mean values. (a-b) The PV zonal and daily anomalies $\left(P_{a}^{* *}\right)$ at day $-15 ;(\mathrm{c}-\mathrm{d})$ the meridional wind zonal and daily anomalies $\left(v_{a}^{*}\right)$ at day $-15 ;(\mathrm{e}-\mathrm{f}) P_{a}^{* *}$ at day $-5 ;(\mathrm{g}-\mathrm{h}) v_{a}^{*}$ at day -5 . (a, c, e, g) show the composites of wave-1 SSWs and (b, d, f, h) show the composites of wave-2 SSWs. 
https://doi.org/10.5194/wcd-2021-14

Preprint. Discussion started: 11 March 2021

(C) Author(s) 2021. CC BY 4.0 License.

Weather and

Climate Dynamics

(c) $\underset{B Y}{(7)}$

advection for wave-1 SSWs increases more slowly but consistently. The rapid growth of the nonlinear process for wave-2 SSWs could be related to a positive feedback between the nonlinear PV flux and $P_{a}^{* *}\left(v_{a}^{*}\right)$ when we tried to interpret the underlying dynamics of the mode equation budget. The linear and nonlinear advection terms are closely linked to the PV flux divergence, while the zonal-mean PV flux can be directly related to the zonal mean momentum budget (McIntyre and Palmer, 1983; Tung, 1986; Plumb, 2010). The zonal-mean poleward PV flux can be further decomposed into the linear and nonlinear components, which play a similar role in the weakening of the polar vortex as the effects of the PV advection terms on the increase of $\frac{d A_{1}}{d t}$. The wave-2 spatial pattern of the linear PV flux helps to precondition the stratospheric basic state and decelerate the polar vortex in the first period of the SSW development for both types of SSWs. When the vortex weakening process evolves to the second period, the evolution of the PV flux for the two types of SSW events bifurcates as the linear and nonlinear PV flux exclusively amplify in the wave- 1 and wave- 2 events, respectively. This bifurcation could be due to the specific evolution of the stratospheric states in the two types of events (Charlton and Polvani, 2007; Albers and Birner, 2014), which can be seen from the horizontal patterns of the PV and meridional wind zonal anomalies. Our results suggest that the high wavenumber pattern that emerged in the second period for wave-2 SSWs is closely connected to the wave- 2 wave flux and could be essential to the split of the vortex.

As suggested by Aikawa et al. (2019), the mode decomposition analysis allows us to investigate the contribution of each EOF mode to the breakdown of the polar vortex, and the way the associated spatial patterns play a role in the temporal evolution of the PC time series. We found that the interactions involving the low modes are the dominant contributors to the weakening of the polar vortex, especially for the increase of the linear advection term in the first period (i.e., day -25 to day -15). Further investigation of the contribution from each EOF mode to the linear and nonlinear advection terms in the mode equation budget suggests that the increase of the linear advection term in the first period is largely influenced by the second and third EOF modes, which both show a wave-1 structure. The first EOF mode only plays an important role at around one week before the SSW event, implying that the process for the vortex weakening is initiated by modes that are not zonally symmetric. In terms of the nonlinear advection terms, the interactions amongst the first five EOF modes are important when approaching the onset of SSWs.

In conclusion, our study finds signals that are representative of SSW events as early as 25 days preceding the events. This lead time is significantly longer than the current deterministic predictability limit of SSWs. We furthermore find that mode decomposition analysis can help to distinguish wave-1 and wave-2 events at least one week ahead of the event, which is longer than previous lead times identified in previous studies (Karpechko, 2018; Taguchi, 2018; Domeisen et al., 2020b). The timescale of emergence of this signal for the distinction between wave- 1 and wave- 2 events provides insights into the different dynamical processes for these two types of SSWs, and thus could be potentially used as a predictor for the SSW type in future studies. Given that the noticeable increase in $\frac{d A_{1}}{d t}$ in the simplified GCM (Isca model) experiment, which directly indicates the weakening of the polar vortex, shows up only around 10 days before the onset of SSWs (i.e., at shorter lead times than for reanalysis), suggests that the observed atmosphere tends to be more predictable than the model, which agrees with theory (Smith et al., 2016; Scaife and Smith, 2018). Applying the mode decomposition analysis to more complex forecasting models, 
https://doi.org/10.5194/wcd-2021-14

Preprint. Discussion started: 11 March 2021

(c) Author(s) 2021. CC BY 4.0 License.

Weather and

Climate Dynamics

Discussions

i.e., S2S reforecast models (Vitart et al., 2017), to examine the predictability of SSWs will provide further insights into the dynamics of polar vortex weakening events and the potential to predict these events beyond the current lead times; this is work in progress.

Code and data availability. The Isca modeling framework was downloaded from the GitHub repository (https://github.com/ ExeClim/Isca) (Vallis et al., 2018)). ERA-Interim reanalysis (Dee et al., 2011) was obtained from the ECMWF server (https://apps.ecmwf.int/datasets/data/interimfull-daily).

\section{Appendix: A. Mode decomposition budget equation}

In this appendix, we show the derivation procedure of obtaining the mode decomposition equation budget (Eq. (4)). The spatial patterns of the projections $\left(\boldsymbol{U}_{1}, \boldsymbol{U}_{2}, \ldots, \boldsymbol{U}_{d}\right)$ of the wind vector daily anomalies $\left(\boldsymbol{V}_{a}\right)$ are computed by projecting $\boldsymbol{V}_{a}$ onto the PC time series $\left(\left\{A_{1}, A_{2}, \ldots, A_{d}\right\}\right)$. Note that $\boldsymbol{U}_{1}, \boldsymbol{U}_{2}, \ldots, \boldsymbol{U}_{d}$ are not necessarily orthogonal. The anomaly terms of PV and the wind vector fields can be written as

$$
\begin{aligned}
P_{a} & =\sum_{n=1}^{d} E_{n} A_{n}, \\
\boldsymbol{V}_{a} & =\sum_{n=1}^{d} \boldsymbol{U}_{n} A_{n} .
\end{aligned}
$$

By substituting Eq. (A1) into Eq. (3), we get

$$
\sum_{n=1}^{d} E_{n} \frac{d A_{n}}{d t}=-\boldsymbol{V}_{c} \cdot \sum_{n=1}^{d} \nabla E_{n} A_{n}-\left(\sum_{n=1}^{d} \boldsymbol{U}_{n} A_{n}\right) \cdot \nabla P_{c}-\sum_{n=1}^{d} \boldsymbol{U}_{n} A_{n} \cdot \sum_{n=1}^{d} \nabla E_{n} A_{n}+F_{a} .
$$

535 Taking the inner product between Eq. (A2) and a given EOF mode $E_{k}$, we obtain

$$
\sum_{n=1}^{d}\left\langle E_{k}, E_{n}\right\rangle \frac{d A_{n}}{d t}=-\sum_{n=1}^{d}\left\langle E_{k}, \boldsymbol{V}_{c} \cdot \nabla E_{n}\right\rangle A_{n}-\sum_{n=1}^{d}\left\langle E_{k}, \boldsymbol{U}_{n} \cdot \nabla P_{c}\right\rangle A_{n}-\sum_{m=1}^{d} \sum_{n=1}^{d}\left\langle E_{k}, \boldsymbol{U}_{m} \cdot \nabla E_{n}\right\rangle A_{m} A_{n}+\left\langle E_{k}, F_{a}\right\rangle .
$$

Given that $\left\{A_{1}, A_{2}, \ldots, A_{d}\right\}$ form an orthogonal basis, i.e., $\left\langle A_{k}, A_{n}\right\rangle=\delta_{k n} C_{k}$ for a given mode $k$ (with $\delta_{k n}=1$ for $n=k$, and $\delta_{k n}=0$ for $n \neq k$ ), with $C_{k}$ being the eigenvalue of mode $k$, the mode equation budget can be computed as

$$
\frac{d A_{k}}{d t}=\frac{1}{C_{k}}\left(-\sum_{n=1}^{d} L_{k n}^{A} A_{n}-\sum_{n=1}^{d} L_{k n}^{B} A_{n}-\sum_{m=1}^{d} \sum_{n=1}^{d} N_{k m n} A_{m} A_{n}+F_{k}\right),
$$

which is the expression of Eq. (4) in Section 2.2. 
https://doi.org/10.5194/wcd-2021-14

Preprint. Discussion started: 11 March 2021

(c) Author(s) 2021. CC BY 4.0 License.

(c) (1)

Weather and

Climate Dynamics

Discussions

\section{Appendix: B. Approximation of $A_{1}$ tendency equation with the PV flux}

In this appendix, we show the derivation for obtaining approximated expression of the linear and nonlinear terms (Eq. (9)) in the $A_{1}$ tendency equation using the PV flux form. Taking the inner product between Eq. (8) and $E_{1}$, and neglecting the variation of $\frac{1}{\rho_{\theta}}$ in the inner products, we obtain the following approximation of the rate of change of $A_{1}$ :

$C_{1} \frac{d A_{1}}{d t} \approx-\frac{1}{\rho_{\theta}} \sum_{n=1}^{d}\left\langle E_{1}, \nabla \cdot\left(\rho_{\theta} E_{n} \boldsymbol{V}_{c}\right)\right\rangle A_{n}-\frac{1}{\rho_{\theta}} \sum_{n=1}^{d}\left\langle E_{1}, \nabla \cdot\left(\rho_{\theta} P_{c} \boldsymbol{U}_{n}\right)\right\rangle A_{n}-$

545

$$
\frac{1}{\rho_{\theta}} \sum_{m=1}^{d} \sum_{n=1}^{d}\left\langle E_{1}, \nabla \cdot\left(\rho_{\theta} E_{n} \boldsymbol{U}_{m}\right)\right\rangle A_{m} A_{n}-\left\langle E_{1}, P \frac{1}{\rho_{\theta}} \frac{\partial \rho_{\theta}}{\partial t}\right\rangle+\left\langle E_{1}, F_{a}\right\rangle,
$$

where $C_{1}$ is the eigenvalue of the first EOF mode of PV. Comparing the linear and nonlinear terms in Eq. (B1) with those in Eq. (A3), we can see that

$L_{1 n}^{A}+L_{1 n}^{B} \approx\left\langle E_{1}, \nabla \cdot\left(\rho_{\theta} P_{a} \boldsymbol{V}_{c}\right)\right\rangle+\left\langle E_{1}, \nabla \cdot\left(\rho_{\theta} P_{c} \boldsymbol{V}_{a}\right)\right\rangle$,

$N_{1 m n} \approx\left\langle E_{1}, \nabla \cdot\left(\rho_{\theta} P_{a} \boldsymbol{V}_{a}\right)\right\rangle$.

As we mentioned in Section 2.3, given the wavenumber-0 structure of $E_{1}$, we further approximate $E_{1}$ to be only a function of latitude. Thus, taking the inner product with $E_{1}$ can be approximated as taking a latitude-weighted integral of the meridional gradient of PV flux as demonstrated below:

$\left\langle E_{1}, \nabla \cdot\left(\rho_{\theta} P_{a} \boldsymbol{V}_{c}\right)\right\rangle+\left\langle E_{1}, \nabla \cdot\left(\rho_{\theta} P_{c} \boldsymbol{V}_{a}\right)\right\rangle \approx 2 \pi a^{2} \int_{\phi_{1}}^{\phi_{2}} E_{1}(\phi) \frac{\partial\left[\rho_{\theta} P_{a}^{* *} v_{c}^{*}\right]+\left[\rho_{\theta} P_{c}^{* *} v_{a}^{*}\right]}{\partial y} \cos \phi d \phi$,

$\left\langle E_{1}, \nabla \cdot\left(\rho_{\theta} P_{a} \boldsymbol{V}_{a}\right)\right\rangle \approx 2 \pi a^{2} \int_{\phi_{1}}^{\phi_{2}} E_{1}(\phi) \frac{\partial\left[\rho_{\theta} P_{a}^{* *} v_{a}^{*}\right]}{\partial y} \cos \phi d \phi$,

where $a$ is the radius of the Earth, $\phi$ is the latitude with $\phi_{1}=30^{\circ} \mathrm{N}$ and $\phi_{2}=90^{\circ} \mathrm{N}$. Using (B3), we can obtain the approximated linear and nonlinear terms as in Eq. (9).

\section{Appendix: C. EOF modes and PC time series of PV in Isca model}

In this appendix, we show the spatial pattern of the first 10 EOF modes of PV anomalies at $850 \mathrm{~K}$ and the associated first PC time series in the Isca model as described in Section 2.1. The first 10 modes together explain $\approx 82 \%$ of the variance of the PV anomalies of all winter days in Isca model. 

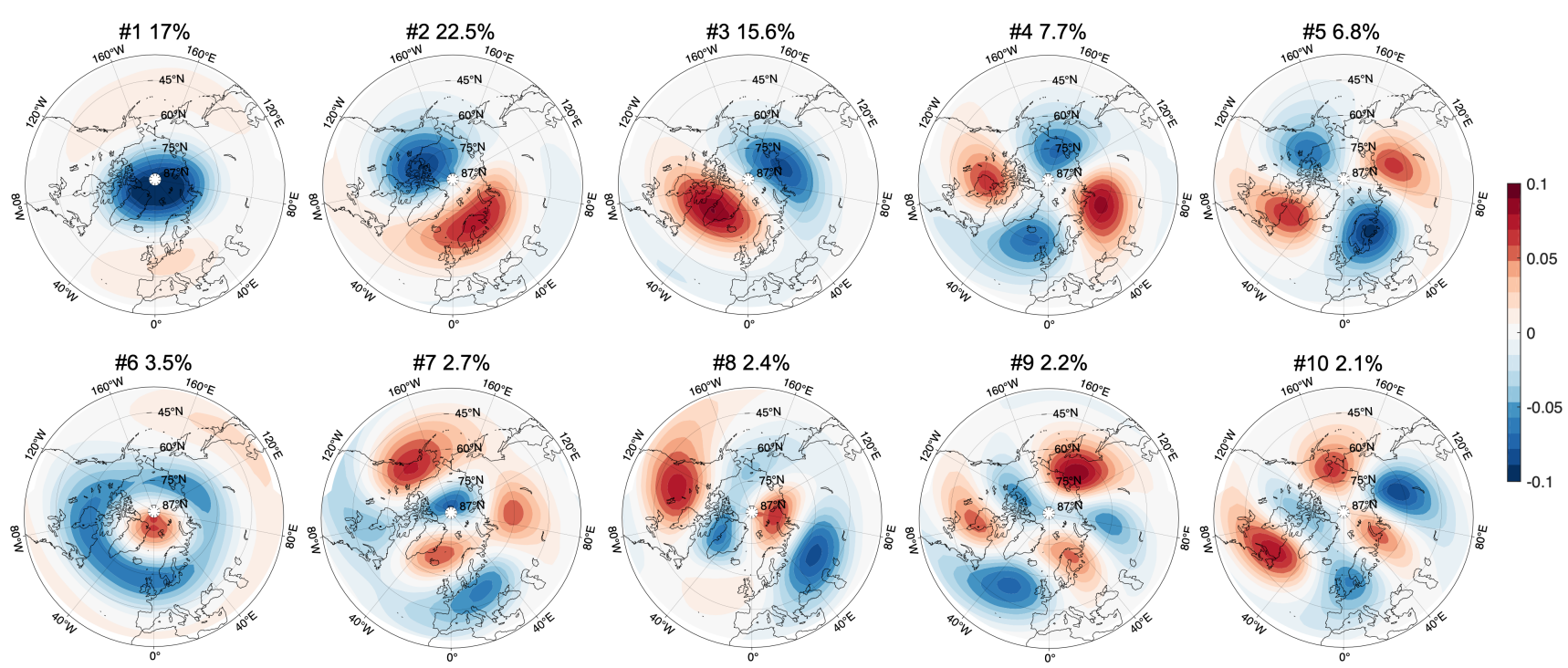

Figure C1. The first 10 EOF spatial patterns of PV at $850 K\left(E_{1}, E_{2}, \ldots, E_{10}\right)$ of the combined set of basis vectors as described in Section 2.1 using Isca-model daily data. The percentage number indicates the variance explained by each EOF.

\section{(a)}

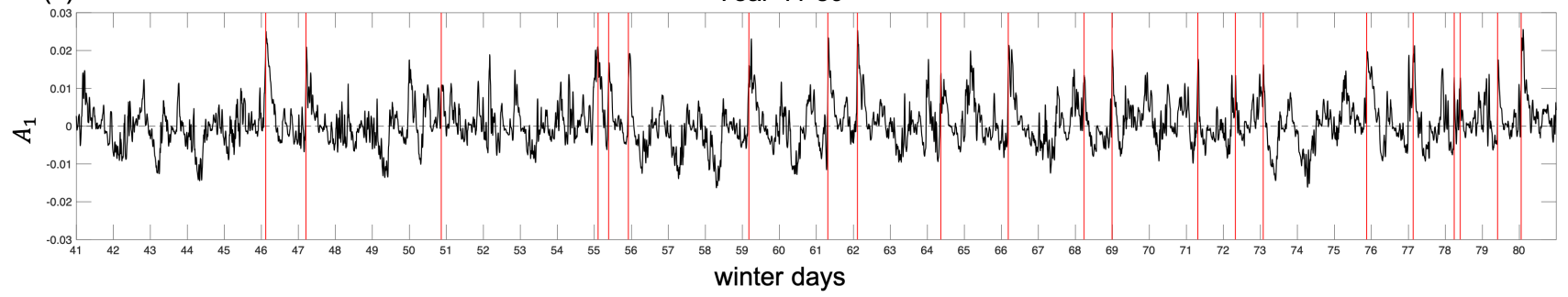

(b)

Year $81-130$

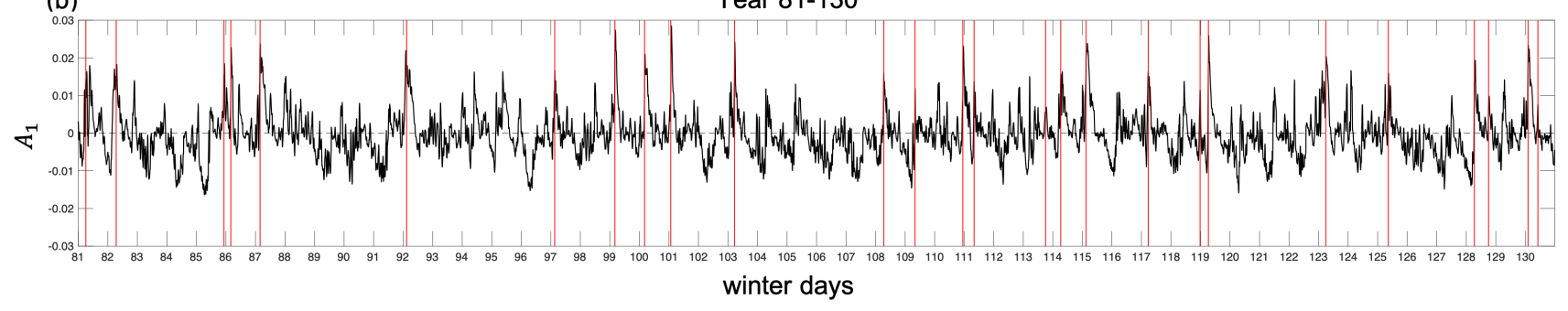

Figure C2. The PC time series $\left(A_{1}\right)$ of winter days corresponding to the first EOF spatial pattern $\left(E_{1}\right)$ using Isca-model winter daily data (from October to April) for (a) Year 41-80 and (b) Year 81-130. The PC time series for winter days of Year 1-40 can be seen in Figure 2b. The red lines indicate the onset dates of SSW events. 
https://doi.org/10.5194/wcd-2021-14

Preprint. Discussion started: 11 March 2021

(c) Author(s) 2021. CC BY 4.0 License.

Weather and

Climate Dynamics

(c) (1)

\section{Appendix: D. Mode decomposition budget for displacement and split SSWs in ERA-interim}

In this appendix, we show the $A_{1}$ mode decomposition budget for the composites of displacement and split SSW events in ERA-interim, which can be compared with Figure 3.
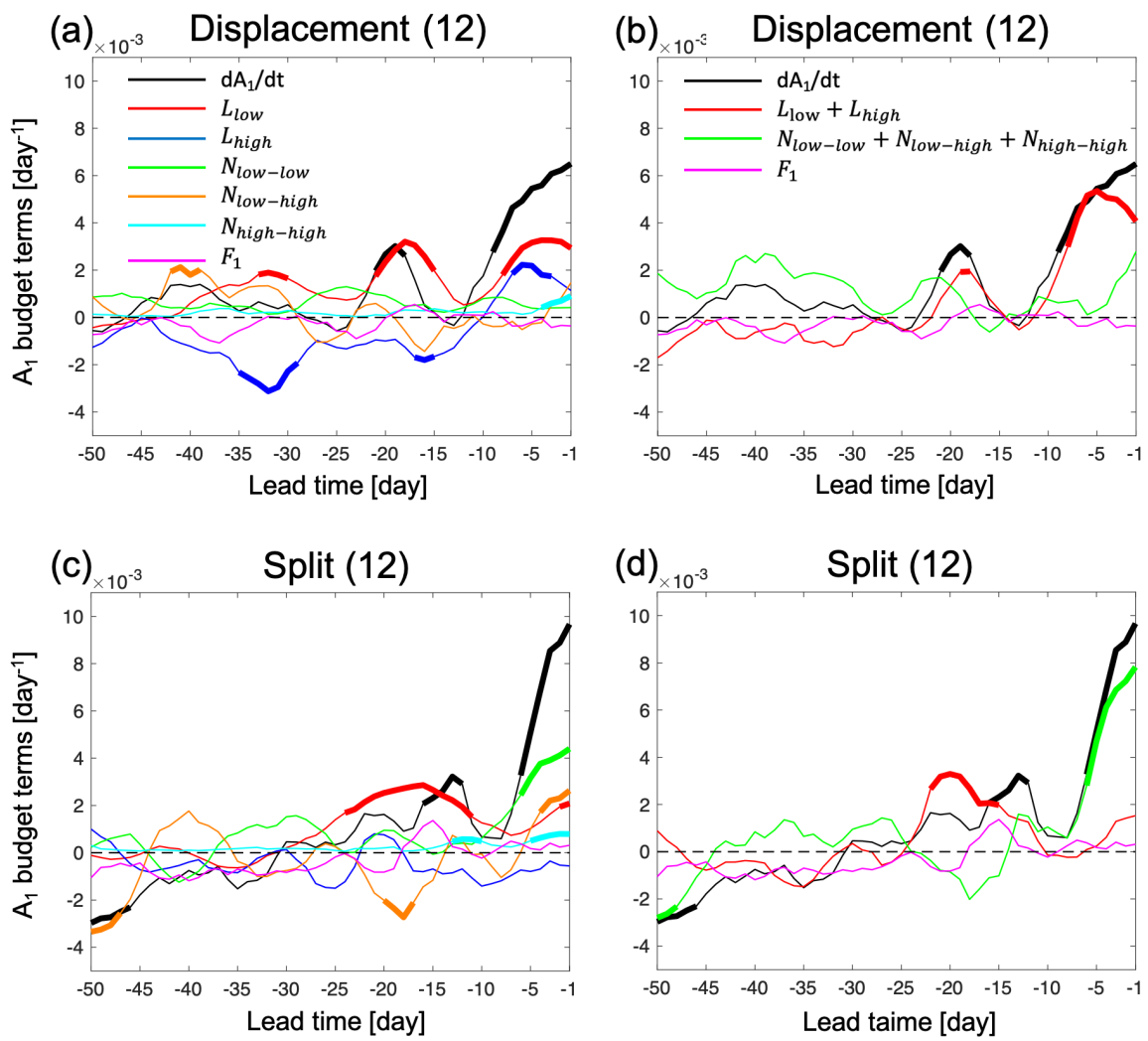

Figure D1. The composite of the $A_{1}$ budget as a function of lead time from 50 to 1 days before the onset of events in ERA-Interim. (a, b) composite of displacement events, (c, d) composite of split events. (a, c) show each term of the mode equation budget (Eq. (5)) separately, and (b, d) show the sum of the linear and nonlinear terms for the different types of events. Number in the bracket in each panel title indicates the number of SSW events. A 5-day running mean is applied to all lines. Bold lines indicate the values that are outside of the 2.5 th to 97.5 th percentile range of normal winter days values from bootstrapping as described in the text. The representation of each line color in (c) and in (d) is the same as the legend in (a) and in (b), respectively.

Author contributions. Z.W. performed the derivations, data analysis, made the figures and wrote the first draft. B.J.-E. performed the Isca model computations. Z.W. and D.D. designed and supervised the study. All authors provided feedback for the manuscript and helped with discussions of the analysis. 
https://doi.org/10.5194/wcd-2021-14

Preprint. Discussion started: 11 March 2021

(C) Author(s) 2021. CC BY 4.0 License.

(c) (i)

Weather and

Climate Dynamics

Discussions

565 Competing interests. The authors declare no competing interests.

Acknowledgements. The work of Z.W. and R.d.F. was funded by the Swiss Data Science Center within the project EXPECT (C18-08).

Funding from the Swiss National Science Foundation to B.J.-E. and D.D. through project PP00P2_170523 is gratefully acknowledged. 
https://doi.org/10.5194/wcd-2021-14

Preprint. Discussion started: 11 March 2021

(C) Author(s) 2021. CC BY 4.0 License.
Weather and

Climate Dynamics

Discussions

\section{References}

Aikawa, T., Inatsu, M., Nakano, N., and Iwano, T.: Mode-decomposed equation diagnosis for atmospheric blocking development, Journal of the Atmospheric Sciences, 76, 3151-3167, https://doi.org/10.1175/JAS-D-18-0362.1, 2019.

Albers, J. R. and Birner, T.: Vortex preconditioning due to planetary and gravity waves prior to sudden stratospheric warmings, Journal of the Atmospheric Sciences, 71, 4028-4054, https://doi.org/10.1175/JAS-D-14-0026.1, 2014.

Ayarzagüena, B., Langematz, U., and Serrano, E.: Tropospheric forcing of the stratosphere: A comparative study of the two different major stratospheric warmings in 2009 and 2010, Journal of Geophysical Research, 116, D18 114, https://doi.org/10.1029/2010JD015023, http: //doi.wiley.com/10.1029/2010JD015023, 2011.

Ayarzagüena, B., Palmeiro, F. M., Barriopedro, D., Calvo, N., Langematz, U., and Shibata, K.: On the representation of major stratospheric warmings in reanalyses, Atmospheric Chemistry and Physics, 19, 9469-9484, https://doi.org/10.5194/acp-19-9469-2019, 2019.

Baldwin, M. P. and Dunkerton, T. J.: Stratospheric harbingers of anomalous weather regimes, Science, 294, 581-584, https://doi.org/10.1126/science.1063315, 2001.

Baldwin, M. P., Ayarzagüena, B., Birner, T., Butchart, N., Butler, A. H., Charlton-Perez, A. J., Domeisen, D. I. V., Garfinkel, C. I., Garny, H., Gerber, E. P., Hegglin, M. I., Langematz, U., and Pedatella, N. M.: Sudden Stratospheric Warmings, Reviews of Geophysics, 59, 1-37, https://doi.org/10.1029/2020rg000708, 2021.

Bancalá, S., Krüger, K., and Giorgetta, M.: The preconditioning of major sudden stratospheric warmings, Journal of Geophysical Research Atmospheres, 117, 1-12, https://doi.org/10.1029/2011JD016769, 2012.

Birner, T. and Albers, J. R.: Sudden stratospheric warmings and anomalous upward wave activity flux, Scientific Online Letters on the Atmosphere, 13, 8-12, https://doi.org/10.2151/sola.13A-002, 2017.

Blume, C., Matthes, K., and Horenko, I.: Supervised learning approaches to classify sudden stratospheric warming events, Journal of the Atmospheric Sciences, 69, 1824-1840, https://doi.org/10.1175/JAS-D-11-0194.1, 2012.

Boljka, L. and Birner, T.: Tropopause-level planetary wave source and its role in two-way troposphere-stratosphere coupling, Weather and Climate Dynamics, 1, 555-575, https://doi.org/10.5194/wcd-1-555-2020, 2020.

Butler, A. H., Seidel, D. J., Hardiman, S. C., Butchart, N., Birner, T., and Match, A.: Defining sudden stratospheric warmings, Bulletin of the American Meteorological Society, 96, 1913-1928, https://doi.org/10.1175/BAMS-D-13-00173.1, 2015.

Charlton, A. J. and Polvani, L. M.: A New Look at Stratospheric Sudden Warmings. Part I: Climatology and Modeling Benchmarks, Journal of Climate, 20, 449-469, https://doi.org/10.1175/JCLI3994.1, http://journals.ametsoc.org/doi/abs/10.1175/JCLI3994.1, 2007.

Charney, J. G. and Drazin, P.: Propagation of Planetary-Scale Disturbances from the Lower into the Upper Atmosphere, Journal of Geophysical Research, 66, 83-109, https://doi.org/https://doi.org/10.1029/JZ066i001p00083, 1961.

Charney, J. G. and Eliassen, A.: A Numerical Method for Predicting the Perturbations of the Middle Latitude Westerlies, Tellus, 1, 38-54, https://doi.org/10.3402/tellusa.v1i2.8500, 1949.

De La Cámara, A., Birner, T., and Albers, J. R.: Are sudden stratospheric warmings preceded by anomalous tropospheric wave activity?, Journal of Climate, 32, 7173-7189, https://doi.org/10.1175/JCLI-D-19-0269.1, 2019.

Dee, D. P., Uppala, S. M., Simmons, A. J., Berrisford, P., Poli, P., Kobayashi, S., Andrae, U., Balmaseda, M. A., Balsamo, G., Bauer, P., Bechtold, P., Beljaars, A. C., van de Berg, L., Bidlot, J., Bormann, N., Delsol, C., Dragani, R., Fuentes, M., Geer, A. J., Haimberger, L., Healy, S. B., Hersbach, H., Hólm, E. V., Isaksen, L., Kållberg, P., Köhler, M., Matricardi, M., Mcnally, A. P., Monge-Sanz, B. M., Morcrette, J. J., Park, B. K., Peubey, C., de Rosnay, P., Tavolato, C., Thépaut, J. N., and Vitart, F.: The ERA-Interim reanalysis: 
https://doi.org/10.5194/wcd-2021-14

Preprint. Discussion started: 11 March 2021

(c) Author(s) 2021. CC BY 4.0 License.

(c) (i)

Weather and

Climate Dynamics

Discussions

Configuration and performance of the data assimilation system, Quarterly Journal of the Royal Meteorological Society, 137, 553-597, https://doi.org/10.1002/qj.828, 2011.

Domeisen, D. I. V.: Estimating the Frequency of Sudden Stratospheric Warming Events From Surface Observations of the North Atlantic Oscillation, Journal of Geophysical Research: Atmospheres, 124, 3180-3194, https://doi.org/10.1029/2018JD030077, 2019.

Domeisen, D. I. V. and Plumb, A. R.: Traveling planetary-scale Rossby waves in the winter stratosphere: The role of tropospheric baroclinic instability, Geophysical Research Letters, 39, 1-5, https://doi.org/10.1029/2012GL053684, 2012.

Domeisen, D. I. V., Butler, A. H., Charlton-Perez, A. J., Ayarzagüena, B., Baldwin, M. P., Dunn-Sigouin, E., Furtado, J. C., Garfinkel, C. I., Hitchcock, P., Karpechko, A. Y., Kim, H., Knight, J., Lang, A. L., Lim, E. P., Marshall, A., Roff, G., Schwartz, C., Simpson, I. R., Son, S. W., and Taguchi, M.: The Role of the Stratosphere in Subseasonal to Seasonal Prediction: 2. Predictability Arising From StratosphereTroposphere Coupling, Journal of Geophysical Research: Atmospheres, 125, 1-20, https://doi.org/10.1029/2019JD030923, 2020a.

Domeisen, D. I. V., Butler, A. H., Charlton-Perez, A. J., Ayarzagüena, B., Baldwin, M. P., Dunn-Sigouin, E., Furtado, J. C., Garfinkel, C. I., Hitchcock, P., Karpechko, A. Y., Kim, H., Knight, J., Lang, A. L., Lim, E. P., Marshall, A., Roff, G., Schwartz, C., Simpson, I. R., Son, S. W., and Taguchi, M.: The Role of the Stratosphere in Subseasonal to Seasonal Prediction: 1. Predictability of the Stratosphere, Journal of Geophysical Research: Atmospheres, 125, 1-17, https://doi.org/10.1029/2019JD030920, 2020b.

Edmon, H. J., Hoskins, B. J., and McIntyre, M. E.: Eliassen-Palm Cross Sections for the Troposphere, https://doi.org/10.1175/15200469(1980)037<2600:EPCSFT>2.0.CO;2, http://journals.ametsoc.org/doi/abs/10.1175/1520-0469\%281980\%29037\%3C2600\% 3AEPCSFT\%3E2.0.CO\%3B2, 1981.

Esler, J. G. and Matthewman, N. J.: Stratospheric sudden warmings as self-tuning resonances. Part II: Vortex displacement events, Journal of the Atmospheric Sciences, 68, 2505-2523, https://doi.org/10.1175/JAS-D-11-08.1, 2011.

Gerber, E. P. and Polvani, L. M.: Stratosphere-Troposphere Coupling in a Relatively Simple AGCM: The Importance of Stratospheric Variability, Journal of Climate, 22, 1920-1933, 2009.

Held, I. M., Ting, M., and Wang, H.: Northern winter stationary waves: Theory and modeling, Journal of Climate, 15, 2125-2144, https://doi.org/10.1175/1520-0442(2002)015<2125:NWSWTA>2.0.CO;2, 2002.

Hitchcock, P. and Haynes, P. H.: Stratospheric control of planetary waves, Geophysical Research Letters, 43, 884-11, https://doi.org/10.1002/2016GL071372, 2016.

Hoskins, B. J., McIntyre, M. E., and Robertson, A. W.: On the use and significance of isentropic potential vorticity maps, Quarterly Journal of the Royal Meteorological Society, 111, 877-946, https://doi.org/10.1002/qj.49711147002, http://doi.wiley.com/10.1002/qj.49711147002, 1985.

Jiménez-Esteve, B. and Domeisen, D. I. V.: Nonlinearity in the North Pacific Atmospheric Response to a Linear ENSO Forcing, Geophysical Research Letters, 46, 2271-2281, https://doi.org/10.1029/2018GL081226, 2019.

Jucker, M.: Are Sudden Stratospheric Warmings Generic? Insights from an Idealized GCM, Journal of the Atmospheric Sciences, 73, 50615080, https://doi.org/10.1175/JAS-D-15-0353.1, http://journals.ametsoc.org/doi/10.1175/JAS-D-15-0353.1, 2016.

Jucker, M. and Reichler, T.: Dynamical Precursors for Statistical Prediction of Stratospheric Sudden Warming Events, Geophysical Research Letters, 45, 124-13, https://doi.org/10.1029/2018GL080691, 2018.

Karpechko, A. Y.: Predictability of sudden stratospheric warmings in the ECMWF extended-range forecast system, Monthly Weather Review, 146, 1063-1075, https://doi.org/10.1175/MWR-D-17-0317.1, 2018.

Karpechko, A. Y., Hitchcock, P., Peters, D. H. W., and Schneidereit, A.: Predictability of downward propagation of major sudden stratospheric warmings, Quarterly Journal of the Royal Meteorological Society, 60, 1459-1470, https://doi.org/10.1002/qj.3017, 2017. 
https://doi.org/10.5194/wcd-2021-14

Preprint. Discussion started: 11 March 2021

(C) Author(s) 2021. CC BY 4.0 License.

(c) (i)

Weather and

Climate Dynamics

Discussions

Kidston, J., Scaife, A. A., Hardiman, S. C., Mitchell, D. M., Butchart, N., Baldwin, M. P., and Gray, L. J.: streams , storm tracks and surface weather, Nature Publishing Group, 8, 433-440, https://doi.org/10.1038/ngeo2424, http://dx.doi.org/10.1038/ngeo2424, 2015.

King, A. D., Butler, A. H., Jucker, M., Earl, N. O., and Rudeva, I.: Observed Relationships Between Sudden Stratospheric Warmings and European Climate Extremes, Journal of Geophysical Research: Atmospheres, 124, 13 943-13 961, https://doi.org/10.1029/2019JD030480, 2019.

Kolstad, E. W., Breiteig, T., and Scaife, A. A.: The association between stratospheric weak polar vortex events and cold air outbreaks in the Northern Hemisphere, Quarterly Journal of the Royal Meteorological Society, 136, 886-893, https://doi.org/10.1002/qj.620, 2010.

Labitzke, K.: Stratospheric-mesospheric midwinter disturbances: A summary of observed characteristics, Journal of Geophysical Research, 86, 9665, https://doi.org/10.1029/jc086ic10p09665, 1981.

Limpasuvan, V., Thompson, D. W., and Hartmann, D. L.: The life cycle of the Northern Hemisphere sudden stratospheric warmings, Journal of Climate, 17, 2584-2596, https://doi.org/10.1175/1520-0442(2004)017<2584:TLCOTN>2.0.CO;2, 2004.

Lindgren, E. A., Sheshadri, A., and Plumb, R. A.: Sudden Stratospheric Warming Formation in an Idealized General Circulation Model Using Three Types of Tropospheric Forcing, Journal of Geophysical Research: Atmospheres, 123, 125-10, https://doi.org/10.1029/2018JD028537, 2018.

Manney, G., Farrara, J., and Mechoso, C.: Simulations of the February 1979 Stratospheric Sudden Warming: Model Comparisons and ThreeDimensional Evolution, Monthly Weather Review, 122, 1115-1140, 1994.

Matsuno, T.: A Dynamical Model of the Stratospheric Sudden Warming, Journal of the Atmospheric Sciences, 28, 1479-1494, https://doi.org/10.1175/1520-0469(1971)028<1479:ADMOTS>2.0.CO;2, 1971.

Matthewman, N. J. and Esler, J. G.: Stratospheric sudden warmings as self-tuning resonances. Part I: Vortex splitting events, Journal of the Atmospheric Sciences, 68, 2481-2504, https://doi.org/10.1175/JAS-D-11-07.1, 2011.

Matthewman, N. J., Esler, J. G., Charlton-Perez, A. J., and Polvani, L. M.: A new look at stratospheric sudden warmings. Part III: Polar vortex evolution and vertical structure, Journal of Climate, 22, 1566-1585, https://doi.org/10.1175/2008JCLI2365.1, 2009.

McIntyre, E.: How Well do we Understand the Dynamics of Stratospheric Warmings?, Journal of the Meteorological Society of Japan, 60, 37-65, https://doi.org/https://doi.org/10.2151/jmsj1965.60.1_37, 1982.

McIntyre, M. E. and Palmer, T.: Breaking planetary waves in the stratosphere, Nature, 305, 593-600, 1983.

Mukougawa, H., Hirooka, T., and Kuroda, Y.: Influence of stratospheric circulation on the predictability of the tropospheric Northern Annular Mode, Geophysical Research Letters, 36, L08 814, https://doi.org/10.1029/2008GL037127, http://doi.wiley.com/10.1029/2008GL037127, 2009.

Nakagawa, K. I. and Yamazaki, K.: What kind of stratospheric sudden warming propagates to the troposphere?, Geophysical Research Letters, 33, 3-6, https://doi.org/10.1029/2005GL024784, 2006.

Plumb, R. A.: Planetary waves and the extratropical winter stratosphere, in: The Stratosphere: Dynamics, Transport, and Chemistry, vol. 190, pp. 23-41, Geophysical Monograph Series, https://doi.org/10.1029/2009GM000888, http://doi.wiley.com/10.1029/2009GM000888, 2010.

Polvani, L. M. and Waugh, D. W.: Upward wave activity flux as a precursor to extreme stratospheric events and subsequent anomalous surface weather regimes, Journal of Climate, 17, 3548-3554, https://doi.org/10.1175/1520-0442(2004)017<3548:UWAFAA>2.0.CO;2, 2004.

Scaife, A. A. and Smith, D.: A signal-to-noise paradox in climate science, npj Climate and Atmospheric Science, 1, https://doi.org/10.1038/s41612-018-0038-4, http://dx.doi.org/10.1038/s41612-018-0038-4, 2018. 
https://doi.org/10.5194/wcd-2021-14

Preprint. Discussion started: 11 March 2021

(C) Author(s) 2021. CC BY 4.0 License.
Weather and

Climate Dynamics

Discussions

Scaife, A. A., Karpechko, A. Y., Baldwin, M. P., Brookshaw, A., Butler, A. H., Eade, R., Gordon, M., Maclachlan, C., Martin, N., Dunstone, N., and Smith, D.: Seasonal winter forecasts and the stratosphere, Atmospheric Science Letters, 17, 51-56, https://doi.org/10.1002/asl.598, 2016.

Scinocca, J. F. and Haynes, P. H.: Dynamical forcing of stratospheric planetary waves by tropospheric baroclinic eddies, Journal of the Atmospheric Sciences, 55, 2361-2392, https://doi.org/10.1175/1520-0469(1998)055<2361:DFOSPW>2.0.CO;2, 1998.

Sigmond, M., Scinocca, J. F., Kharin, V. V., and Shepherd, T. G.: Enhanced seasonal forecast skill following stratospheric sudden warmings, Nature Geoscience, 6, 98-102, https://doi.org/10.1038/ngeo1698, http://dx.doi.org/10.1038/ngeo1698, 2013.

Smith, D. M., Scaife, A. A., Eade, R., and Knight, J. R.: Seasonal to decadal prediction of the winter North Atlantic Oscillation: Emerging capability and future prospects, Quarterly Journal of the Royal Meteorological Society, 142, 611-617, https://doi.org/10.1002/qj.2479, 2016.

Taguchi, M.: Comparison of Subseasonal-to-Seasonal Model Forecasts for Major Stratospheric Sudden Warmings, Journal of Geophysical Research: Atmospheres, 123, 231-10, https://doi.org/10.1029/2018JD028755, 2018.

Tung, K. K.: Nongeostrophic Theory of Zonally Averaged Circulation. Part I: Formulation, Journal of The Atmospheric Science, 43, 2600$2618,1986$.

695 Vallis, G. K., Colyer, G., Geen, R., Gerber, E., Jucker, M., Maher, P., Paterson, A., Pietschnig, M., Penn, J., and Thomson, S. I.: Isca, v1.0: A framework for the global modelling of the atmospheres of Earth and other planets at varying levels of complexity, Geoscientific Model Development, 11, 843-859, https://doi.org/10.5194/gmd-11-843-2018, 2018.

Vitart, F., Ardilouze, C., Bonet, A., Brookshaw, A., Chen, M., Codorean, C., Déqué, M., Ferranti, L., Fucile, E., Fuentes, M., Hendon, H., Hodgson, J., Kang, H. S., Kumar, A., Lin, H., Liu, G., Liu, X., Malguzzi, P., Mallas, I., Manoussakis, M., Mastrangelo, D., MacLachlan, C., McLean, P., Minami, A., Mladek, R., Nakazawa, T., Najm, S., Nie, Y., Rixen, M., Robertson, A. W., Ruti, P., Sun, C., Takaya, Y., Tolstykh, M., Venuti, F., Waliser, D., Woolnough, S., Wu, T., Won, D.-J., Xiao, H., Zaripov, R., and Zhang, L.: The Subseasonal to Seasonal (S2S) Prediction Project Database, Bulletin of the American Meteorological Society, 98, 163-173, 2017. 